\title{
Antibody-mediated delivery of VEGF-C potently reduces chronic skin inflammation
}

\author{
Simon Schwager, Silvana Renner, Teresa Hemmerle, Sinem Karaman, Steven T. Proulx, \\ Roman Fetz, Alexandra Michaela Golding-Ochsenbein, Philipp Probst, Cornelia Halin, Dario Neri, \\ and Michael Detmar
}

Institute of Pharmaceutical Sciences, Swiss Federal Institute of Technology, ETH Zurich, Zurich, Switzerland.

\begin{abstract}
VEGF-C is an important mediator of lymphangiogenesis and has been shown to alleviate chronic inflammation in a variety of disease models. In this study, we investigated whether targeted delivery of VEGF-C to sites of inflammation and site-specific activation of lymphatic vessels would represent a clinically feasible strategy for treating chronic skin inflammation. To this end, we generated a fusion protein consisting of human VECF-C fused to the F8 antibody (F8-VECF-C), which is specific for the alternatively spliced, angiogenesis-marking extradomain A (EDA) of fibronectin. In two mouse models of psoriasis-like skin inflammation, mediated by transgenic VECF-A overexpression or repeated application of imiquimod, intravenous treatment with F8VEGF-C but not with untargeted VEGF-C significantly reduced ear skin edema and was as effective as the clinically used TNF- $\alpha$ receptor-Fc fusion protein (TNFR-Fc). Treatment with F8-VEGF-C led to a marked expansion of lymphatic vessels in the inflamed skin and significantly improved lymphatic drainage function. At the same time, treatment with F8-VEGF-C significantly reduced leukocyte numbers, including $\mathrm{CD}^{+}$and $\gamma \delta$ T cells. In sum, our results reveal that targeted delivery of VEGF-C and site-specific induction of lymphatic vessels represent a potentially new and promising approach for the treatment of chronic inflammatory diseases.
\end{abstract}

Authorship note: SS and SR contributed equally to this work.

Conflict of interest: DN is a cofounder and shareholder of Philogen, a biotech company that owns and is developing derivatives of $\mathrm{F} 8$ antibodies for clinical applications. MD, CH, TH, DN, SR, and SS are inventors on a pending patent for F8-VEGF-C (PCT/EP2018/064900)

License: Copyright 2018, American Society for Clinical Investigation.

Submitted: September 11, 2018 Accepted: October 24, 2018 Published: December 6, 2018

\section{Reference information:} JCI Insight. 2018;3(23):e124850. https://doi.org/10.1172/jci. insight.124850.

\section{Introduction}

The lymphatic system plays an important role in tissue fluid homeostasis, dietary lipid absorption, and immune surveillance. Lymphatic vessels are heavily involved in regulating the inflammatory response by draining extravasated fluid, antigens, inflammatory mediators, and antigen-presenting cells from the periphery to the lymph nodes, where specific immune responses are mounted (1). In inflamed tissue, the lymphatic vasculature undergoes extensive remodeling and expansion, including lymphangiogenesis (the formation of new lymphatic vessels) and enlargement of preexisting vessels. One of the major and best-characterized growth factors involved in these processes is VEGF-C, which signals mainly via VEGFR-3 (2), a receptor specifically expressed by lymphatic endothelial cells (LECs) but not by blood vascular endothelial cells under physiological conditions (3). Importantly, previous studies from our laboratory and from others have revealed that transgenic or virus-mediated delivery of VEGF-C potently alleviated chronic skin inflammation (4), rheumatoid arthritis (5), and inflammatory bowel disease (6) in experimental mouse models. Conversely, blocking VEGF-C signaling led to disease aggravation in mouse models of psoriasis (4), chronic arthritis (7), inflammatory bowel disease (8), and airway inflammation (9). While activation of the lymphatic vasculature is therefore beneficial in the setting of chronic inflammation, there are currently no drugs available that specifically activate lymphatic vessels, making VEGF-C the prime candidate to fill this urgent need.

Despite the evidence for the therapeutic potential of VEGF-C for the treatment of chronic inflammatory diseases, clinical application has been hampered by the lack of practical delivery strategies. Previous studies have focused on transgenic or viral overexpression or local injections of VEGF-C protein for chronic inflammatory diseases. While adequate for research purposes, virus treatments have been associated with an increased risk for malignancies, and topical application is challenging in cases where the disease occurs at multiple sites simultaneously or in locations with restricted accessibility (e.g., the gastrointestinal tract). In addition, for several recombinant growth factors, systemic injections have not resulted in resounding therapeutic success $(10,11)$ and are problematic due to the often wide-spread expression of 
cognate receptors in different tissues, which leads to toxicities and challengingly small therapeutic windows. These problems are additionally exacerbated by the short circulatory half-life of unmodified growth factors, necessitating high doses and repeated administration.

We hypothesized that targeted, antibody-mediated delivery of VEGF-C specifically to the sites of inflammation after systemic application might circumvent these limitations and improve the therapeutic index. Indeed, pharmacodelivery of antibody-cytokine fusion proteins, e.g., incorporating IL-2 or IL-10, has yielded promising results for the therapy of cancer and inflammatory pathologies, such as rheumatoid arthritis and chronic inflammatory bowel disease (reviewed in refs. 12, 13).

Inflammation site-specific delivery of VEGF-C requires a target that is selectively expressed in the inflamed tissues. Fibronectin, an important component of the extracellular matrix, undergoes alternative splicing, and its extradomain A (EDA) is virtually absent under physiological conditions but is incorporated into fibronectin during conditions of tissue remodeling and angiogenesis, as typically observed in cancer and inflammation $(14,15)$. Indeed, EDA has been demonstrated to be a viable target in several disease models, including inflammatory bowel disease (16) and chronic skin inflammation (17). The F8 antibody and fragments thereof are specific for EDA (14), and, due to the favorable expression pattern of their cognate antigen, we hypothesized that F8 constructs might be well suited for targeted delivery of VEGF-C to inflamed tissue.

In this study, we describe the production, biodistribution, activity, and therapeutic potential of the fully human fusion proteins F8-VEGF-C and F8-VEGF-C156Ser, a mutated version that only activates VEGFR-3, in mouse models of chronic inflammatory skin disease. To this end, we induced a chronic skin inflammation in mice overexpressing VEGF-A in the skin (18) and, in a second model, in mice with imiquimod-induced psoriasis-like skin inflammation (19). Systemic therapy with F8-VEGF-C fusion protein induced a marked expansion of the lymphatic network in the inflamed skin, alleviated inflammation-associated skin edema, improved lymphatic clearance function, and reduced inflammatory cell infiltration compared with appropriate therapy control groups.

Thus, this study identifies the first therapeutic protein for the targeted, antibody-mediated delivery of VEGF-C to sites of inflammation to our knowledge. The fusion protein format is convenient for clinical application and may be used for the treatment of chronic inflammatory diseases.

\section{Results}

F8-VEGF-C and F8-VEGF-C156Ser fusion proteins retain antigen affinity and biological activity. We aimed to produce and investigate fusion proteins for the inflammation site-specific delivery of wild-type VEGF-C and of VEGF-C156Ser, a mutant lacking affinity for VEGFR-2, to investigate VEGFR-3-specific therapeutic effects (20)

Human VEGF-C or VEGF-C156Ser was genetically linked to the F8 diabody, yielding the two fusion proteins F8-VEGF-C and F8-VEGF-C156Ser. The diabody format was chosen since it displays improved tissue penetration and faster clearance compared with full antibodies, while maintaining good target-site retention (21). The F8 diabody consists of 2 single-chain variable fragments with a 5-amino acid linker between the variable heavy $\left(\mathrm{V}_{\mathrm{H}}\right)$ and light $\left(\mathrm{V}_{\mathrm{L}}\right)$ domains, leading to the formation of a stable noncovalent homodimeric diabody. The VEGF-C or VEGF-C156Ser was attached to the C-terminus of the F8 diabody via a flexible 15-amino acid linker (Figure 1A). Similarly, VEGF-C was linked to the KSF diabody specific for hen-egg lysozyme (22), making the resulting KSF-VEGF-C an untargeted construct in the mouse organism and an appropriate pharmacokinetic control (nucleotide sequences included in Supplemental Data; supplemental material available online with this article; https://doi.org/10.1172/jci.insight.124850DS1).

The purity and folding of the fusion proteins was assessed by SDS-PAGE and size-exclusion chromatography (Figure 1, B and C, and Supplemental Figure 1, A and B), showing the proteins to be pure and mostly present as covalent dimers under nonreducing conditions, with clear bands around $75 \mathrm{kDa}$ (dimers) and $37 \mathrm{kDa}$ (monomers). Since fusing proteins may influence the structure and functionality of the individual components, we next tested the antigen affinity of the targeting moiety and the biological activity of the VEGF-C and VEGF-C156Ser.

Surface plasmon resonance analysis revealed that the F8 diabody moiety retained its binding affinity for the fibronectin EDA, with dissociation constants $\left(K_{D}\right)$ of $0.48 \mathrm{nM}$ (F8-VEGF-C) and $2 \mathrm{nM}$ (F8-VEGF-C156Ser, Figure 1D). As expected, KSF-VEGF-C did not bind to EDA (Supplemental Figure 1C). The serum half-life of F8-VEGF-C was determined by collecting blood at different time points after intravenous injection of $50 \mu \mathrm{g}$ fusion protein and calculated to be 9.1 minutes (Supplemental Figure 1D). 
A
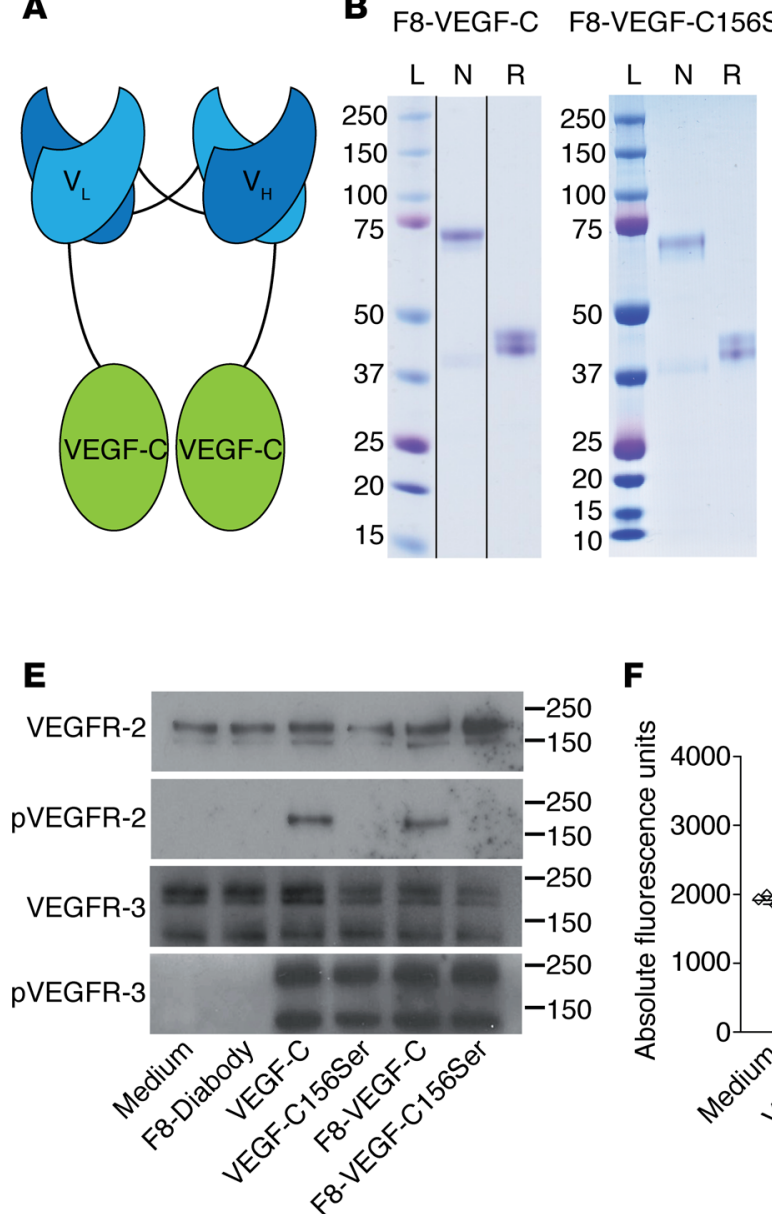

G

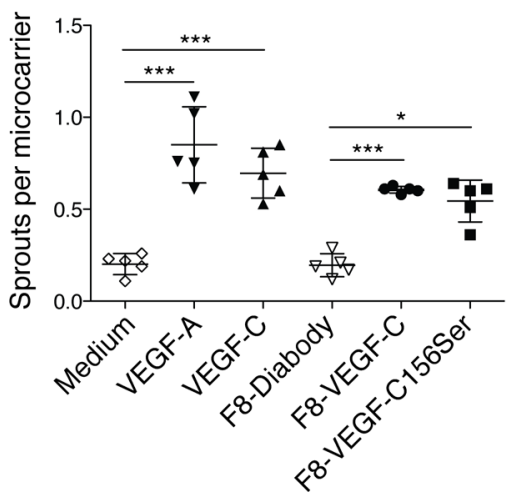

F8-VEGF-C
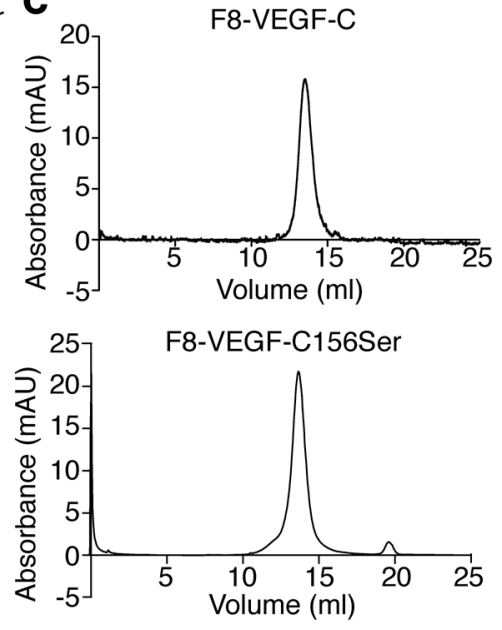

I
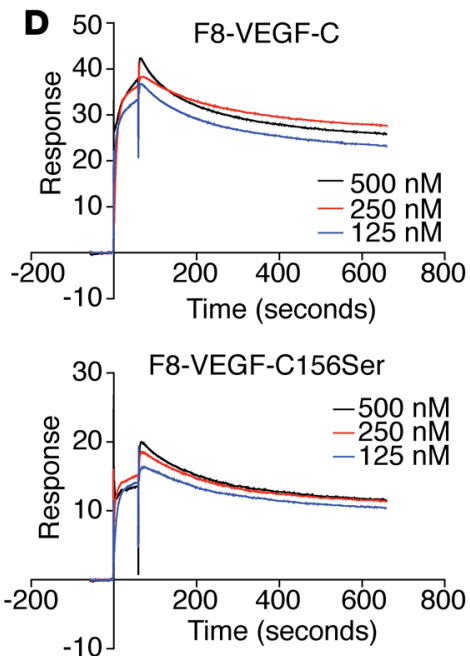

F

H
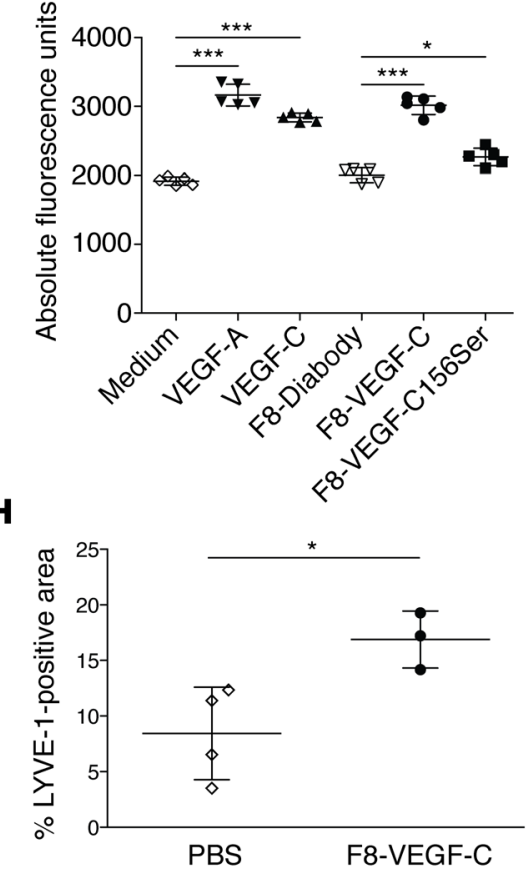

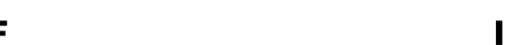

I PBS

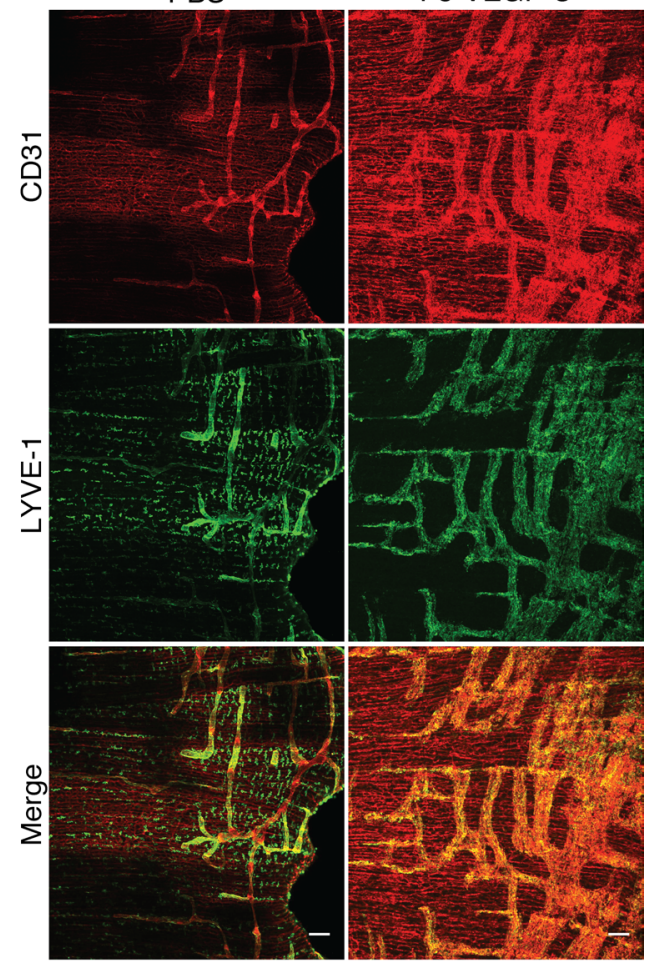

Figure 1. F8-VEGF-C and F8-VEGF-C156Ser fusion proteins retain antigen affinity and biological activity. (A) Schematic representation of the F8-VEGF-C fusion protein. (B) SDS-PAGE analysis of F8-VEGF-C (noncontiguous lanes on same gel) and F8-VEGF-C156Ser. Lanes show size ladder (L) and protein under nonreducing (N) or reducing (R) conditions. (C) Size-exclusion chromatograms of F8-VEGF-C and F8-VEGF-C156Ser. (D) Surface plasmon resonance analysis of F8-VEGF-C and F8-VEGF-C156Ser using an EDA-coated chip. (E) Western blot analysis of VEGFR-3-overexpressing PAE cells for VEGFR-2 and phosphorylated VEGFR-2 as well as VEGFR-3 and phosphorylated VEGFR-3. Molecular weight markers are indicated on the right. (F) Proliferation assay of human LECs after 72 hours of indicated treatment ( $n=5$ wells per condition, 1 -way ANOVA with Bonferroni post test, 1 of 3 similar experiments shown). (G) Sprouting assay of human LECs, counting sprouts formed per LEC-coated bead ( $n=5$ wells per condition, 1-way ANOVA with Bonferroni post test, 1 of 3 similar experiments shown). (H) Quantification of LYVE-1+ area on stained diaphragm from pups (see I, $n=3-4$ animals, 2-tailed Student's $t$ test, 1 of 2 similar experiments shown). (I) Whole-mount immunofluorescence staining for CD31 (red) and LYVE-1 (green) on diaphragms of pups having received 5 injections of PBS or F8-VEGF-C. Scale bar: $100 \mu \mathrm{m}$. Data represent mean \pm SD. ${ }^{*} P$ $<0.05,{ }^{* * *} P<0.001$. 
We next assessed the biological activity of F8-VEGF-C and F8-VEGF-C156Ser in several in vitro assays in comparison to recombinant human VEGF-C. In a first step, Western blot-based phosphorylation analysis was performed to investigate whether the fusion proteins could still signal through VEGFR-2 and/or VEGFR-3. Indeed, in porcine aortic endothelial (PAE) cells transfected to overexpress VEGFR-3 (23), both F8-VEGF-C and F8-VEGF-C156Ser caused tyrosine phosphorylation of VEGFR-3 to a similar degree as free human VEGF-C (Figure 1E). In addition, F8-VEGF-C, but not F8-VEGF-C156Ser, induced phosphorylation of VEGFR-2, which is in line with the specificity of VEGF-C156Ser for VEGFR-3.

All fusion proteins significantly increased proliferation of human LECs to an extent comparable to molar equivalents of free VEGF-C (Figure 1F and Supplemental Figure 1E). F8-VEGF-C and F8-VEGF-C156Ser also significantly promoted sprouting of LECs (Figure 1G).

To study the activity of the fusion proteins in vivo, neonatal FVB mice received daily intraperitoneal injections of F8-VEGF-C, KSF-VEGF-C, or PBS from day 1 to day 5 after birth, causing a strong lymphangiogenic response in the diaphragm. Whole-mount stainings revealed the formation of large LYVE-1 ${ }^{+}$ sheets covering almost the entire pleural side of the diaphragmatic muscle. The LYVE- $1^{+}$area was significantly increased in animals receiving F8-VEGF-C or KSF-VEGF-C compared with PBS-treated mice (Figure 1, H and I, and Supplemental Figure 1, F and G).

These findings indicate that the fusion proteins can be efficiently produced and retain a high-antigen affinity as well as the potent prolymphangiogenic effects of VEGF-C and VEGF-C156Ser in vitro and in vivo.

EDA is expressed in human psoriatic skin and inflamed skin of two mouse models but is absent in healthy skin. In order to achieve inflammation site-specific delivery of VEGF-C, it is essential that the antigen recognized by the targeting moiety is present in inflamed, but largely absent in uninflamed, tissues. As the overarching objective is to utilize the fusion proteins for the therapy of human patients, we first analyzed EDA expression in human psoriatic and healthy skin. Analysis of samples costained for EDA and the vessel marker vascular endothelial cadherin (VE-cadherin) did not reveal any EDA signal in healthy human skin. In psoriatic skin, however, we observed prominent EDA expression around dermal vessels (Figure 2A). In a next step, we checked whether available mouse models of skin inflammation would accurately replicate the observed EDA expression pattern.

We therefore investigated EDA expression in inflamed and noninflamed ear skin of K14-VEGF-A mice. These mice develop a chronic skin inflammation after repeated exposure to the contact sensitizer oxazolone (contact hypersensitivity [CHS]) (18). Indeed, immunofluorescence analysis revealed high EDA expression around vessels in the dermis of chronically inflamed ears (Figure 2B). In contrast, EDA was notably absent in uninflamed ears. These findings could be reproduced in a second model of psoriasis-like skin inflammation, the imiquimod model $(19,24)$. C57BL/6 mice received daily applications on the ears of a cream containing the TLR7/8 agonist imiquimod for 5 consecutive days, followed by 1 day without treatment and a final application on day 7. EDA was detected around vessels in the inflamed tissue but was not expressed in noninflamed ears (Supplemental Figure 2A).

The observed expression pattern in human skin makes EDA a promising target in psoriasis, and this could be replicated in established experimental models of psoriasis-like skin inflammation.

F8-VEGF-C and F8-VEGF-C156Ser selectively accumulate in the inflamed skin of two mouse models. Radioiodinated preparations of F8-VEGF-C and F8-VEGF-C156Ser were intravenously injected in K14-VEGF-A or wild-type FVB mice, and biodistribution was quantitatively assessed after 24 hours to evaluate the inflammation-targeting performance of the constructs. A specific accumulation of F8-VEGF-C and F8-VEGF-C156Ser was observed in inflamed compared with uninflamed ear skin (Figure 2C). Most importantly, in mice undergoing skin inflammation, the fusion proteins targeted the inflamed ear skin, not the uninflamed back skin. A fraction of the injected fusion proteins was found in spleen, kidney, intestine, and lung. Accumulation of fusion proteins in the inflamed ears could also be confirmed by autoradiography (Figure 2D).

Similarly, autoradiography in imiquimod-inflamed ears provided further evidence for the targeting of F8 constructs to inflamed ear tissue (Supplemental Figure 2, B and C). These data demonstrate that F8 fusion proteins preferentially accumulate in inflamed tissue.

F8-VEGF-C and F8-VEGF-C156Ser significantly reduce ear edema and cause expansion of the lymphatic vasculature in CHS-induced skin inflammation. Next, we assessed the therapeutic potential of the fusion proteins in a CHS-induced model of chronic skin inflammation. Hemizygous K14-VEGF-A mice were sensitized with oxazolone and challenged with oxazolone 5 days later on both ears, leading to chronic skin inflammation 
A
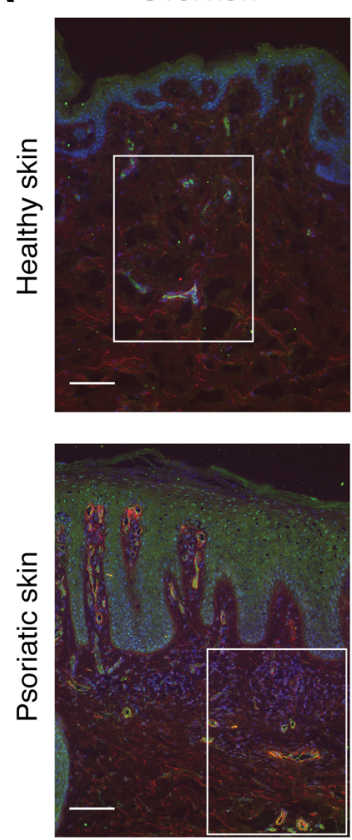

B
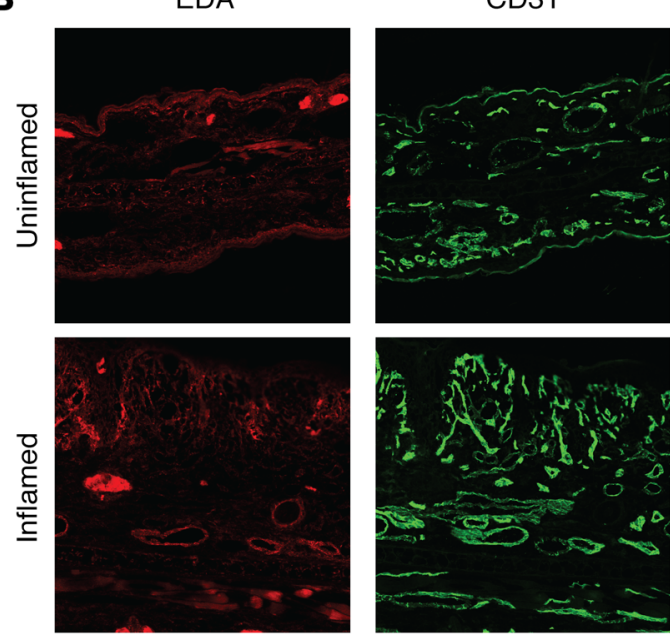

D

Uninflamed

Inflamed

EDA
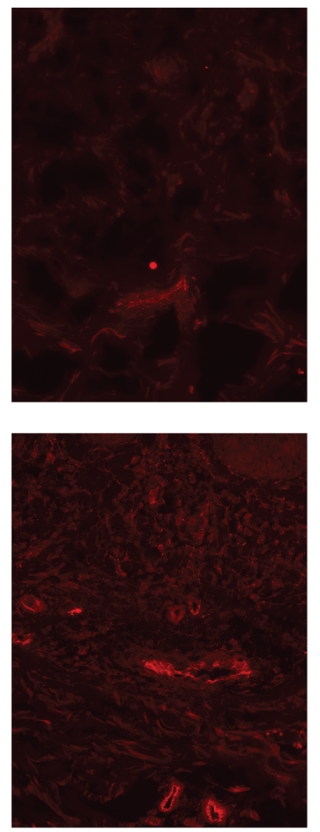

CD31

F8-VEGF-C

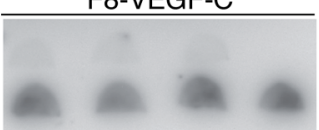

Uninflamed

Inflamed
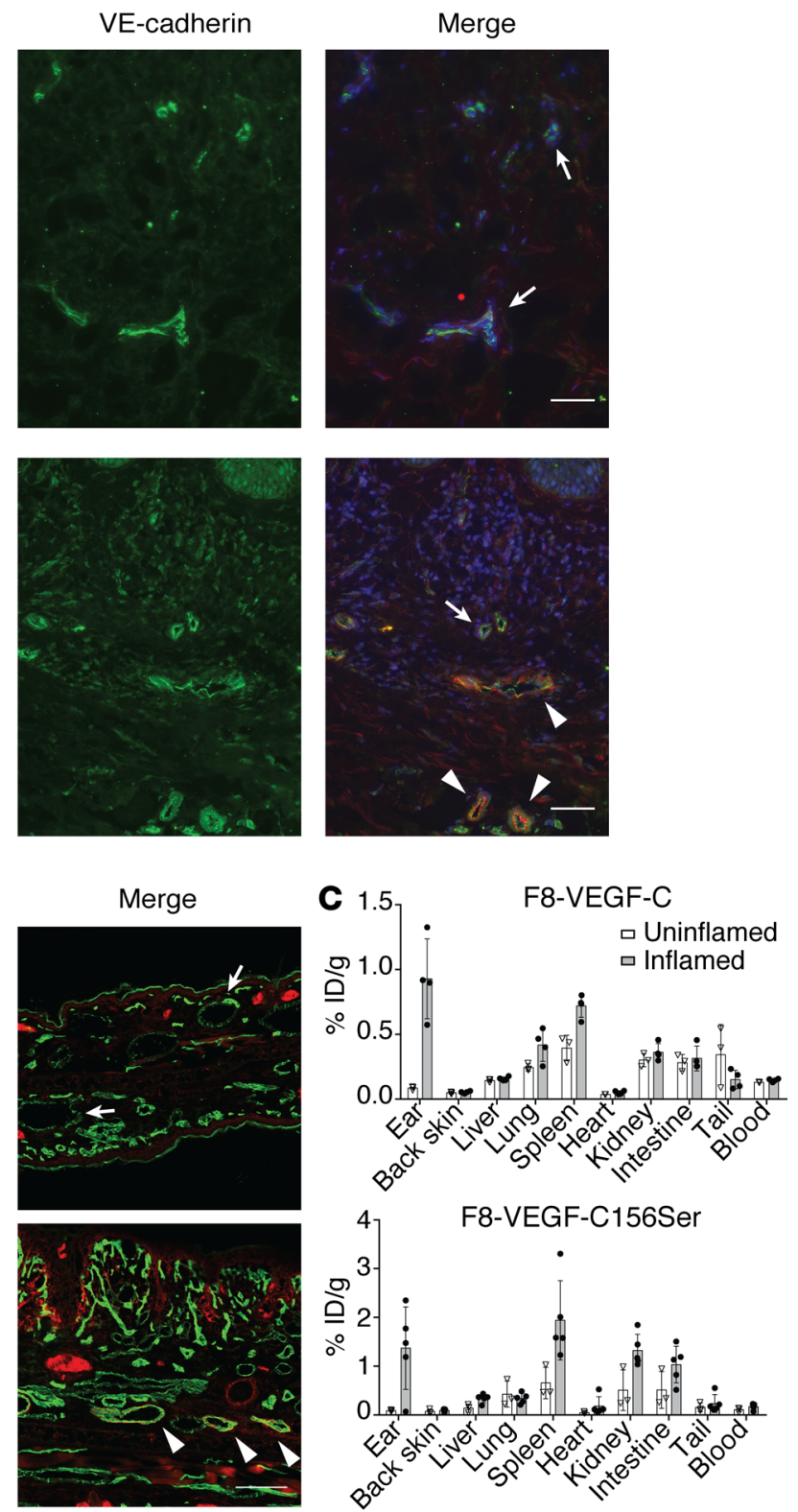

F8-VEGF-C156Ser

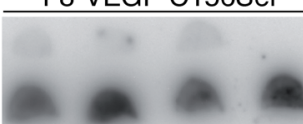

Figure 2. EDA is expressed in human psoriatic lesions and inflamed skin of K14-VEGF-A mice but not in healthy skin, and F8 fusion proteins accumulate in inflamed ear skin. (A) Immunofluorescence stainings for EDA (red) and VE-cadherin (green) in healthy and psoriatic human skin. White boxes highlight enlarged region. Scale bar: $100 \mu \mathrm{m}$ (overview); $50 \mu \mathrm{m}$ (merge). (B) Immunofluorescence stainings for EDA (red) and CD31 (green) in uninflamed and inflamed ear skin of K14-VEGF-A mice 21 days after challenge. Scale bar: $100 \mu \mathrm{m}$. (C) Quantitative biodistribution studies of radioiodinated F8-VEGF-C and F8-VEGF-C156Ser in mice with uninflamed or inflamed ears, expressed as a percentage of injected dose per gram of tissue ( $n=3-5$ mice per group, data represent mean \pm SD). (D) Autoradiography of F8-VEGF-C and F8-VEGF-C156Ser comparing accumulation in inflamed compared with uninflamed ears ( $n=4$ mice per group). ID, injected dose. Arrows indicate EDA- vessels; arrowheads indicate EDA ${ }^{+}$vessels.

and ear swelling (Figure 3A). To evaluate the importance of targeted delivery of VEGF-C for the antiinflammatory properties of the fusion proteins, we compared the effects of the targeted F8-VEGF-C and the untargeted KSF-VEGF-C fusion proteins on ear inflammation. Starting 7 days after challenge, mice received intravenous injections of F8-VEGF-C, KSF-VEGF-C, or PBS every second day, and ear thickness was measured until mice were euthanized on day 15 after challenge (Figure 3A). F8-VEGF-C significantly reduced ear edema by day 11 after challenge, 4 days after initiation of the treatment, while KSF-VEGF-C and PBS 
A

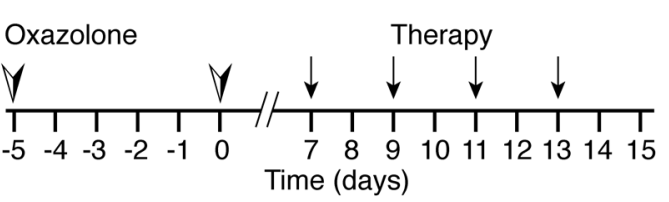

B

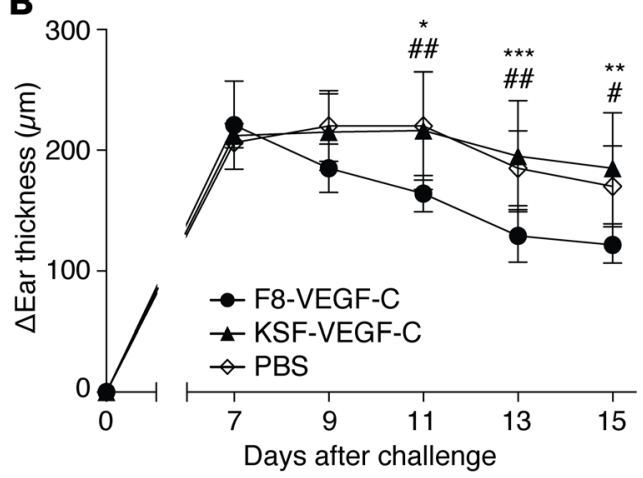

C

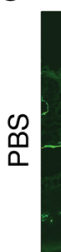

LYVE-1

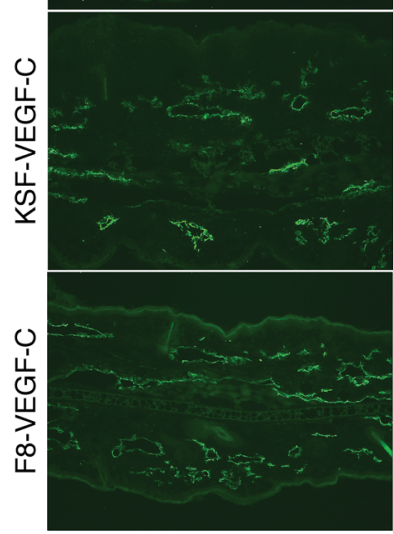

F

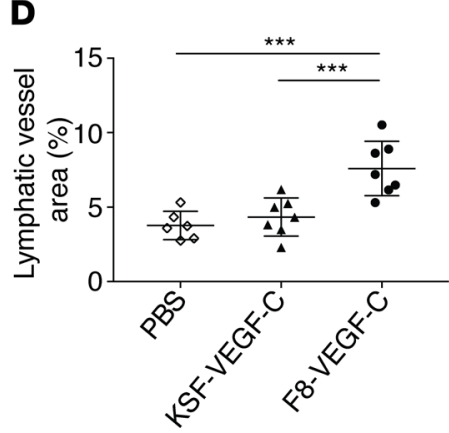

E

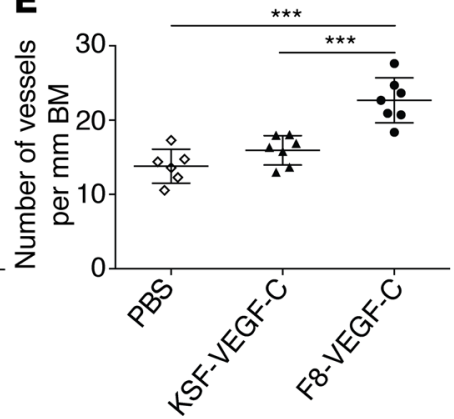

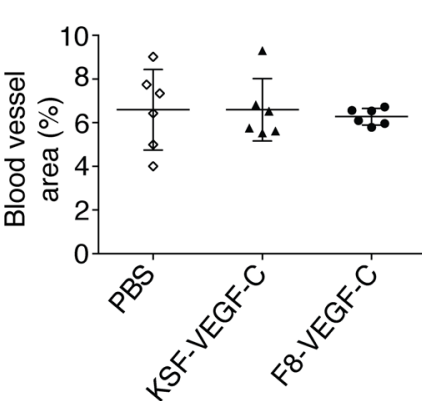

MECA-32

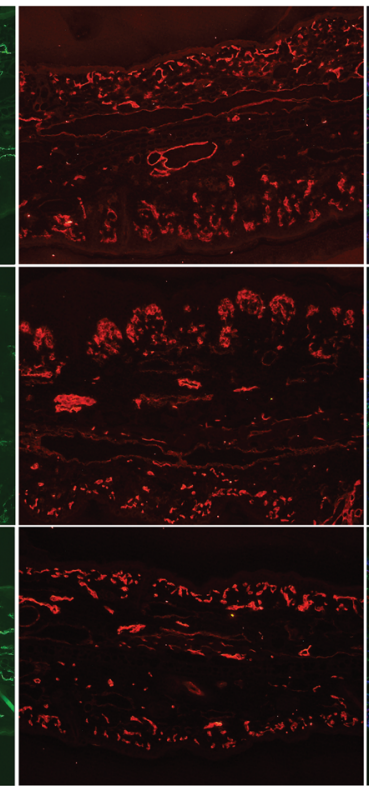

Merge

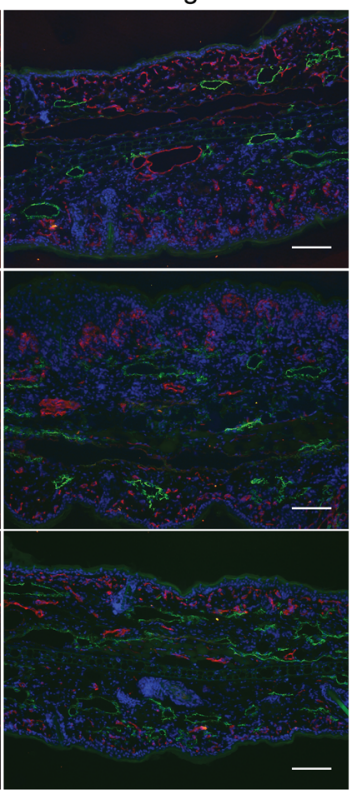

G

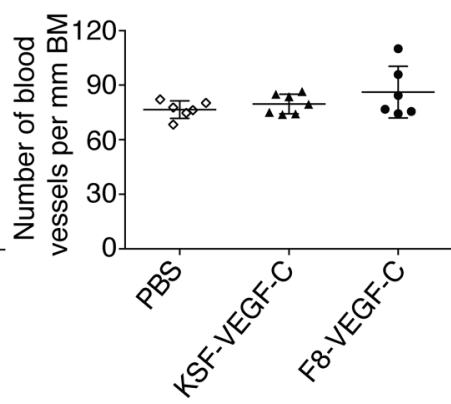

Figure 3. F8-VEGF-C but not KSF-VEGF-C causes lymphatic expansion and alleviates ear inflammation in K14-VEGF-A mice. (A) Schematic of inflammation study time course and treatment schedule. (B) Ear thickness represented as changes compared with ear thickness prior to challenge ( $n=6-7$ mice, 2-way ANOVA with Bonferroni post test, 1 of 2 similar experiments shown; $P$ values noted by an asterisk show comparisons between F8-VEGF-C and KSFVEGF-C and those noted by pound signs show comparisons between F8-VEGF-C and PBS). (C) Immunofluorescence images of ears from mice that received PBS, KSF-VEGF-C, or F8-VEGF-C stained for LYVE-1 (green), MECA-32 (red), and DAPI (blue). Scale bar: $100 \mu \mathrm{m}$. (D) Quantification of Iymphatic vessel area (expressed as percentage of analyzed area, $n=6-7$ animals per group, 1-way ANOVA with Bonferroni post test). (E) Number of Iymphatic vessels in inflamed ears of mice that received the indicated treatment (normalized to basement membrane length, $n=6-7$ animals per group, 1 -way ANOVA with Bonferroni post test). (F) Quantification of blood vessel area in inflamed ears of mice that received the indicated treatment (expressed as percentage of analyzed area, $n=6$ animals per group, 1-way ANOVA with Bonferroni post test). (G) Number of blood vessels in inflamed ears (normalized to basement membrane, $n=6-7$ animals per group). BM, basement membrane. Data represent mean $\pm \mathrm{SD}$. ${ }^{*} P<0.05,{ }^{* *} P<0.01,{ }^{* * *} P<0.001$.

did not affect ear swelling (Figure 3B). To investigate the effect of the treatments on the vasculature, we performed immunofluorescence analyses for the blood vessel-specific marker MECA-32 and the lymphatic vessel marker LYVE-1 in inflamed ear tissue (Figure 3C). We detected a significant increase in total lymphatic vessel area and number upon application of F8-VEGF-C but not KSF-VEGF-C or PBS (Figure 3, D and E). Additionally, lymphatic vessels tended to be enlarged (Supplemental Figure 3A). None of the treatments had an effect on total blood vessel area, number, or size (Figure 3, F and G, and Supplemental Figure 3B).

In our next study, we aimed to compare the antiinflammatory effects of targeted VEGF-C to TNF- $\alpha$ receptor-Fc fusion protein (TNFR-Fc), which is clinically employed for the therapy of rheumatoid arthritis and psoriasis. As before, mice received F8-VEGF-C, F8-VEGF-C156Ser, TNFR-Fc, SIP-F8 (a targeted construct without VEGF-C), or PBS every second day, while ear thickness was measured daily and mice were euthanized 15 days after challenge. SIP-F8 served as a control for both F8-VEGF-C and F8-VEGF-C156Ser, while PBS was the control for TNFR-Fc. TNFR-Fc, F8-VEGF-C, and F8-VEGF-C156Ser significantly reduced ear edema compared with their respective controls (Supplemental Figure 3C). Notably, the fusion proteins reduced ear swelling to a similar degree as the positive control TNFR-Fc employed at a dose previously established to be 
active (25). Immunofluorescence analysis revealed a significant increase in lymphatic vessel area in mice treated F8-VEGF-C or F8-VEGF-C156Ser compared with SIP-F8 (Supplemental Figure 3, D and E). Moreover, F8-VEGF-C induced a trend toward increased lymphatic vessel number (Supplemental Figure 3F). TNFR-Fc had no effect on the lymphatic vasculature. These results indicate that targeted VEGF-C delivery is able to alleviate disease severity in the K14-VEGF-A model of chronic skin inflammation.

Targeted delivery of VEGF-C accelerates resolution of imiquimod-induced skin inflammation. To study the therapeutic potential of targeted VEGF-C delivery in a second mouse model, we used the imiquimod-induced skin inflammation model, in which the mice received daily applications of the TLR7/8 agonist imiquimod on the ears for 7 days (with the exception of day 6) (Figure 4A). This model represents a more acute/subchronic inflammation but reproduces key immunological aspects of human psoriasis (19).

As in the CHS model, we wanted to assess the importance of targeted, as opposed to untargeted, delivery of VEGF-C by comparing F8-VEGF-C to KSF-VEGF-C. SIP-F8 served as an additional control. Starting on day 7, when the inflammation was well established, animals received 3 intravenous injections of F8-VEGF-C, KSF-VEGF-C, or SIP-F8 every other day before being analyzed on day 13 (Figure 4A). F8-VEGF-C significantly accelerated ear edema resolution compared with SIP-F8 and KSF-VEGF-C (Figure 4B). Quantification of MECA-32 and LYVE-1 stainings showed a significant increase of total lymphatic vessel area, number, and size in animals receiving targeted VEGF-C (Figure 4, C-E, and Supplemental Figure 4A). Neither F8-VEGF-C nor KSF-VEGF-C affected the blood vasculature (Figure 4, F and G, and Supplemental Figure 4B).

To assess the therapeutic efficacy of the fusion proteins compared with TNFR-Fc, mice received 3 intravenous injections of F8-VEGF-C, F8-VEGF-C156Ser, TNFR-Fc, SIP-F8, or PBS every second day before being analyzed on day 13. F8-VEGF-C, F8-VEGF-C156Ser, and TNFR-Fc significantly reduced ear thickness compared with SIP-F8 and PBS, respectively (Supplemental Figure 4C). As in the K14-VEGF-A model, both fusion proteins were as effective as the positive control TNFR-Fc. Moreover, quantitative image analysis of inflamed ear skin showed that treatment with F8-VEGF-C, F8-VEGF-C156Ser, and TNFR-Fc caused a marked reduction in epidermal thickness compared with SIP-F8 and PBS, respectively (Supplemental Figure 4, D and E). Immunofluorescence analysis of ear sections stained for MECA-32 and LYVE-1 revealed a significant increase in lymphatic vessel size in animals receiving F8-VEGF-C and, to a smaller degree, in animals injected with F8-VEGF-C156Ser (Supplemental Figure 4, F and G). No change in the number of lymphatic vessels and the number or size of blood vessels was observed (Supplemental Figure 4, H-J). These data demonstrate that the therapeutic effects of targeted VEGF-C delivery could be reproduced in a second disease model. F8-VEGF-C and F8-VEGF-C156Ser both displayed similar efficacy in ear edema resolution, and neither of them caused any expansion of the blood vasculature in the inflamed tissue. We therefore focused on the wild-type F8-VEGF-C in further experiments.

F8-VEGF-C improves lymphatic clearance function. F8-VEGF-C caused a striking expansion of the lymphatic vasculature in the inflamed tissue. However, increased vascular density does not necessarily correlate with improved function. In order to assess whether the observed expansion was reflected in an enhanced lymphatic clearance function, we injected the exclusively lymphatic-drained near-infrared dye P20D800 into the inflamed ears of mice (26). Tracking the signal decay over a series of images after the injection (Figure 5A), we calculated the clearance rate and the tissue half-life of the tracer, thereby estimating the lymphatic clearance function (27). Mice treated with F8-VEGF-C presented with a significantly increased clearance rate and correspondingly decreased tissue half-life compared with PBS-treated animals (Figure 5, $\mathrm{B}$ and $\mathrm{C}$ ), indicating improved lymphatic clearance after F8-VEGF-C application.

F8-VEGF-C causes significant reduction of immune cells in inflamed ear skin. We next investigated the influence of F8-VEGF-C treatment on immune cell populations in the inflamed ears of K14-VEGF-A-transgenic mice. Quantification of ear tissue sections stained for CD45 revealed a small but significant decrease in the $\mathrm{CD}_{4} 5^{+}$cell area (Figure 6A). In addition, there was a reduction in the $\mathrm{CD}^{+}$area (Figure 6B), while macrophages, quantified as CD68 ${ }^{+}$cell area, were not affected by F8-VEGF-C (Figure 6C). Since there was no obvious change in macrophages, we focused on the $\mathrm{T}$ cell compartment and proceeded to perform more detailed analyses by flow cytometry. We were especially interested in changes in the numbers of $\gamma \delta \mathrm{T}$ cells, as these cells have been shown to play a pivotal role in skin inflammation (28). Inflamed ears of K14-VEGF-A mice undergoing CHS were collected at day 15 after challenge and stained for CD45, CD4, CD8, and $\gamma \delta \mathrm{T}$ cell receptor ( $\gamma \delta \mathrm{TCR}$ ). Live cells were gated for CD45 positivity and T cell subpopulations were identified (Supplemental Figure 5) and quantified. In agreement with our immunofluorescence results, we observed 
A
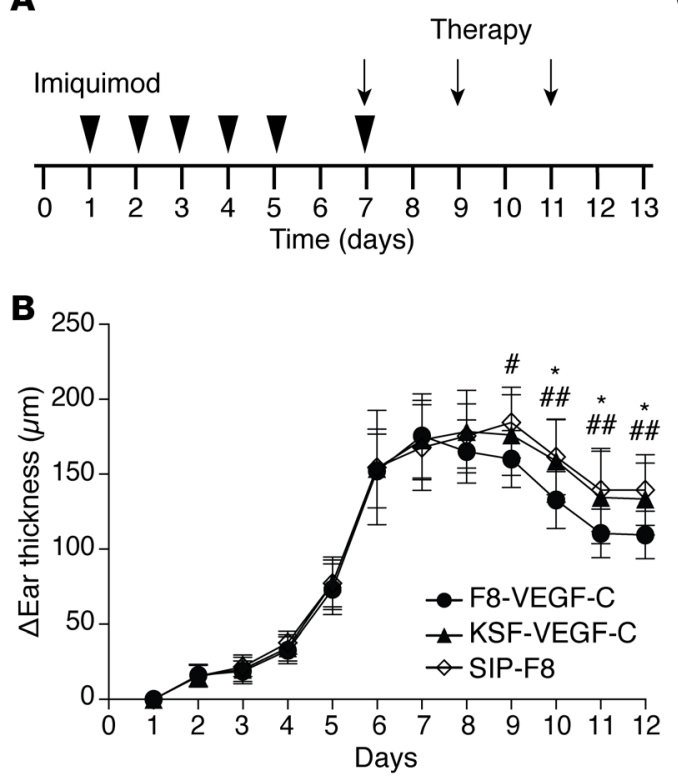

D

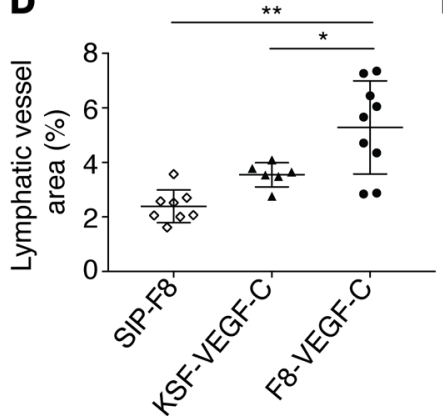

C

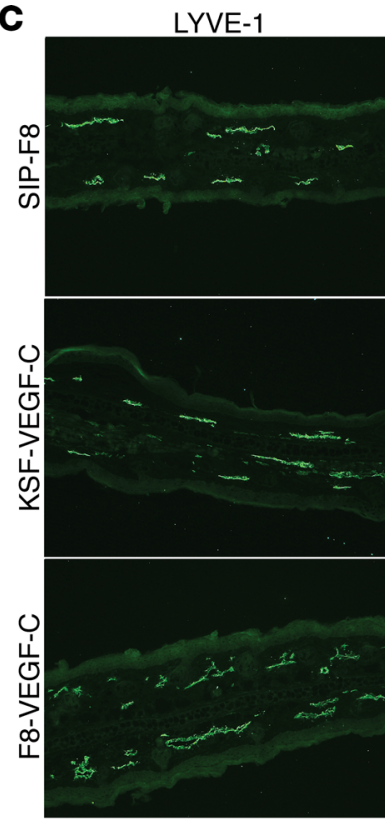

$\mathbf{F}$

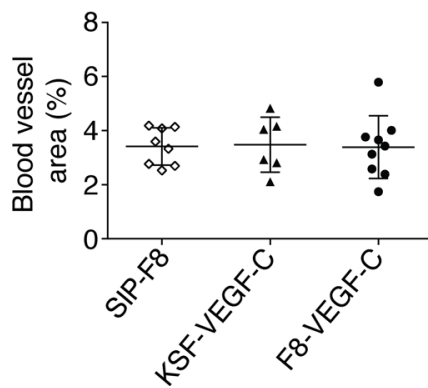

MECA-32

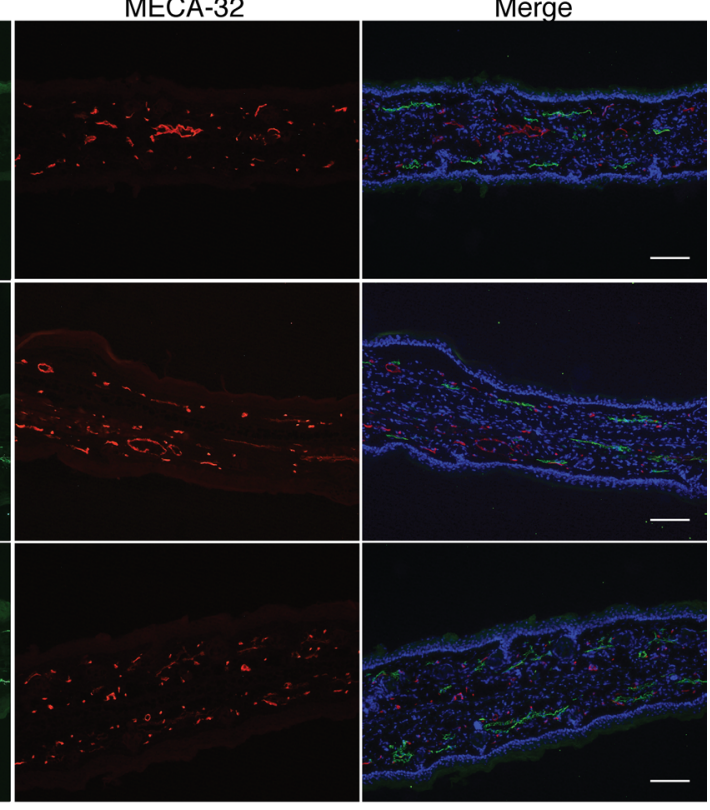

G

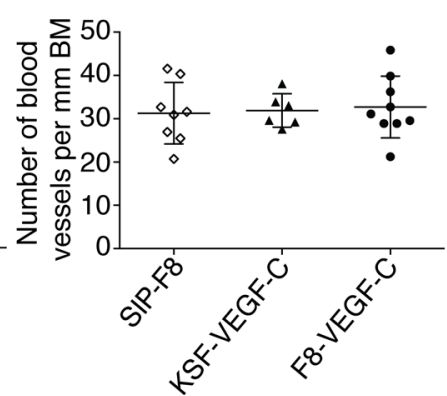

Figure 4. F8-VEGF-C but not KSF-VEGF-C causes lymphatic expansion and accelerates edema resolution in imiquimod-induced inflammation. (A) Schematic of inflammation study time course and treatment schedule. (B) Ear thickness represented as change compared with ear thickness on day 1 ( $n$ = 9 animals per group, 2-way ANOVA with Bonferroni post test; $P$ values noted by an asterisk show comparisons between F8-VEGF-C and KSF-VEGF-C and those noted by pound signs show comparisons between F8-VEGF-C and SIP-F8). (C) Immunofluorescence images of ears from mice that received SIP-F8, KSF-VEGF-C, or F8-VEGF-C stained for LYVE-1 (green), MECA-32 (red), and DAPI (blue). Scale bar: 100 um. (D) Quantification of lymphatic vessel area (expressed as percentage of analyzed area, $n=6-9$ animals per group, 1-way ANOVA with Games-Howell post test). (E) Number of lymphatic vessels in inflamed ears of mice that received the indicated treatment (normalized to basement membrane length, $n=6-9$ animals per group, 1 -way ANOVA with Bonferroni post test). (F) Quantification of blood vessel area in inflamed ears of mice that received the indicated treatment (expressed as percentage of analyzed area, $n=6-9$ animals per group, 1-way ANOVA with Bonferroni post test). (G) Number of blood vessels in inflamed ears (normalized to basement membrane, $n=6-9$ animals per group). Data represent mean $\pm \mathrm{SD}$. BM, basement membrane. ${ }^{*} P<0.05,{ }^{* *} P<0.01,{ }^{* * *} P<0.001$.

a significant decrease in $\mathrm{CD} 45^{+}$as well as $\mathrm{CD} 4^{+}$cells (Figure 6, D and E). Furthermore, the numbers of $\gamma \delta$ $\mathrm{TCR}^{+}$cells were significantly decreased upon treatment with F8-VEGF-C (Figure 6F). This reduction in $\gamma \delta$ $\mathrm{T}$ cells provides a possible explanation for the antiinflammatory effects of the fusion protein.

\section{Discussion}

In this study, we reveal the therapeutic potential of inflammation site-specific delivery of VEGF-C and VEGF-C156Ser linked to the F8 diabody in two mouse models of skin inflammation. Furthermore, we identify a mechanism for the antiinflammatory effects of the F8-VEGF-C fusion protein.

Fusing two proteins represents a major structural modification and may be detrimental to the biological activity of the individual components. However, the F8-targeting moiety retained its antigen affinity, as demonstrated by surface plasmon resonance, and the growth factors were able to induce phosphorylation of VEGFR-2 and VEGFR-3 (F8-VEGF-C) or solely VEGFR-3 (F8-VEGF-C156Ser). Both F8 constructs increased proliferation and sprouting of LECs in established in vitro assays. In addition, the fusion proteins were able to induce lymphatic expansion in the diaphragms of postnatal mice following intraperitoneal 
A

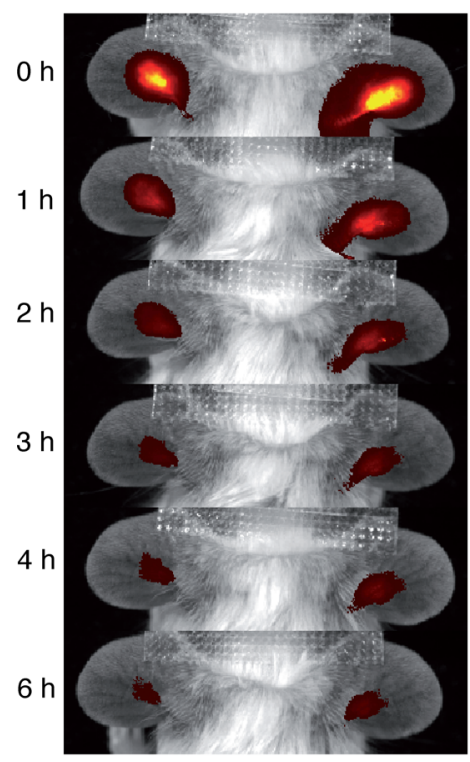

F8-VEGF-C

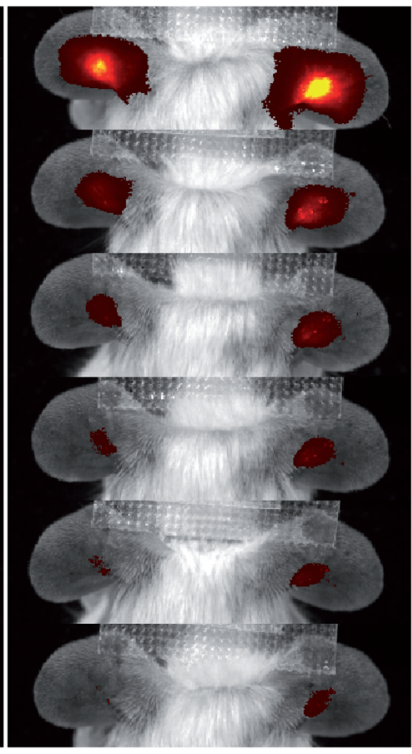

Epi-fluorescence 20‘000

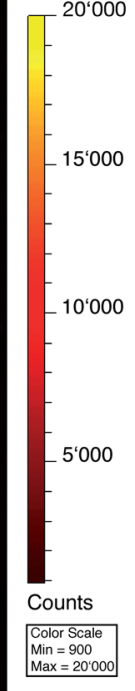

B

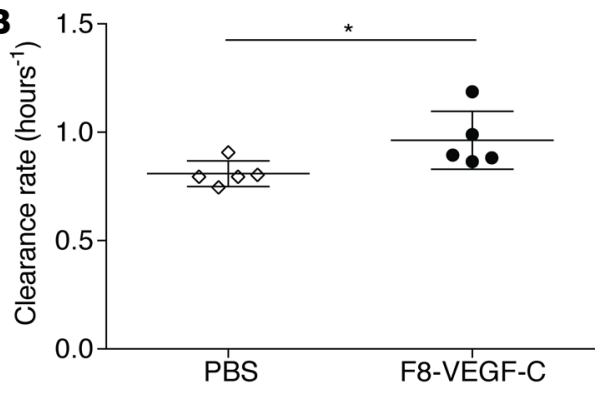

C

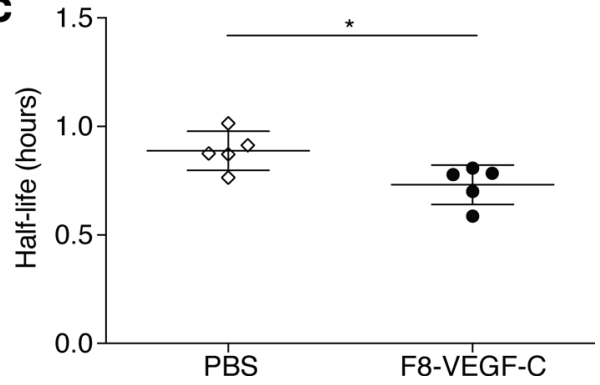

Figure 5. F8-VEGF-C improves lymphatic clearance function. (A) In vivo imaging system (IVIS) images showing tracked signal decay of intradermally injected, exclusively lymphatic-drained near-infrared dye P20D800 in ears of inflamed K14-VEGF-A-transgenic mice that received either PBS or F8-VECF-C. (B) Quantification of clearance rate of P20D800 of inflamed ear skin. (C) Quantification of half-life of P20D800 in inflamed ear skin ( $n=5$ animals per group, 2-tailed Student's $t$ test, 1 of 2 similar experiments shown). Data represent mean \pm SD. ${ }^{*} P<0.05$.

injections, demonstrating that the biological activity was maintained in vivo. Collectively, these results indicate that the targeting moiety retained its antigen affinity and the biological activity of VEGF-C was not impaired after fusion to the F8 or KSF diabody.

A key aspect of therapeutic antibody constructs is their biodistribution profile, which depends on various parameters, such as antigen expression, application route, vessel permeability, and size of the antibody construct. In accordance with previous reports, we found EDA, the antigen of F8 constructs, to be upregulated in the inflamed skin of mice and mostly located around vascular structures (15). Most importantly, this pattern reflects the expression observed in human psoriatic skin, supporting the concept of using F8 constructs for targeted delivery in human patients. Quantitative biodistribution studies of radiolabeled, intravenously injected F8-VEGF-C and F8-VEGF-C156Ser in K14-VEGF-A-transgenic mice revealed a striking enrichment of both fusion proteins in the inflamed compared with uninflamed ear skin. In addition, signal accumulation in the kidney is attributed to blood filtration and urinary clearance of the fusion protein or its metabolites. The biodistribution pattern is in line with previous reports using F8-based constructs $(15,25)$. The potent increase in fusion protein accumulation in inflamed compared with healthy ear skin combined with an overall favorable biodistribution pattern support the F8 diabody as a valid targeting moiety for therapeutic VEGF-C delivery, in agreement with its inflammation specificity in other diseases, such as in rheumatoid arthritis (29).

To assess the therapeutic efficacy, we employed a mouse model with chronic VEGF-A overexpression under control of the keratin-14 promoter. After sensitization and challenge with a contact sensitizer, hemizygous mice develop a chronic skin inflammation, which replicates some characteristics commonly associated with psoriasis-like epidermal thickening (acanthosis), skin reddening, immune cell infiltration in dermis and epidermis, and IL-23a upregulation $(4,18,30)$. Treatments were started 7 days after challenge in the chronic phase of the inflammation. Targeted VEGF-C constructs significantly reduced ear edema after 1 to 2 injections. These findings are in line with previous studies reporting improved CHS skin inflammation resolution and normalization of skin phenotype in mice receiving local injections of VEGF-C156Ser protein and in K14-VEGF-C mice with skin-specific, transgenic overexpression of VEGF-C (4). In an alternative model, intradermal injections of VEGF-C156Ser alleviated UVB radiation-induced ear edema (31). Interestingly, systemically administered untargeted VEGF-C had no positive effect on inflammation severity, indicating that the targeting is essential for the fusion protein's therapeutic effect. Most importantly, the effect of F8-VEGF-C or its mutated form on ear thickness was comparable to TNFR-Fc, which is 
A

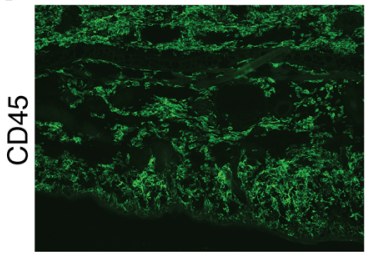

B

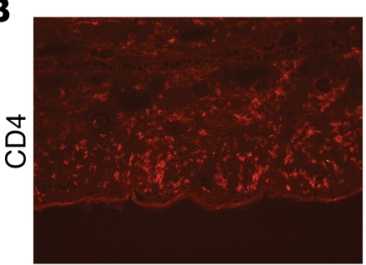

C

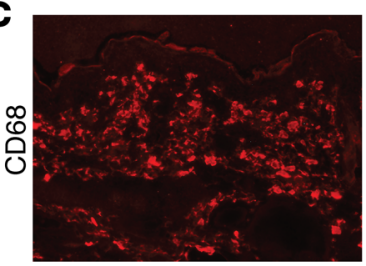

D

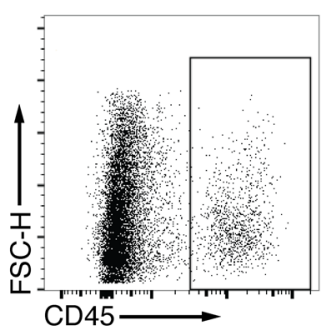

$\mathbf{E}$

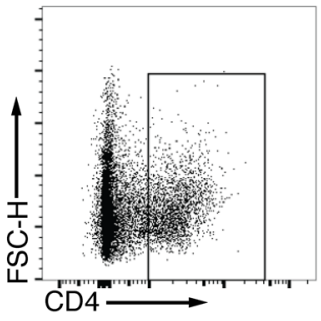

$\mathbf{F}$

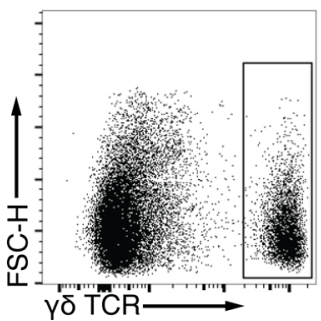

F8-VEGF-C
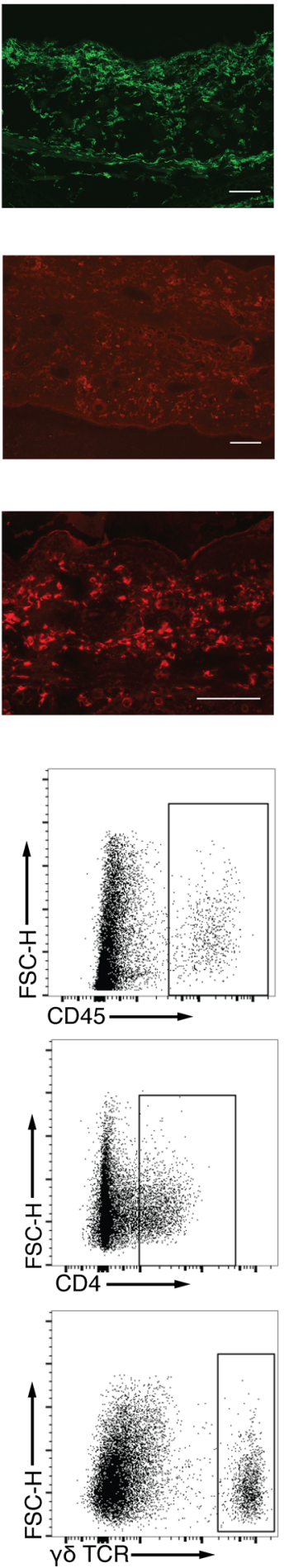
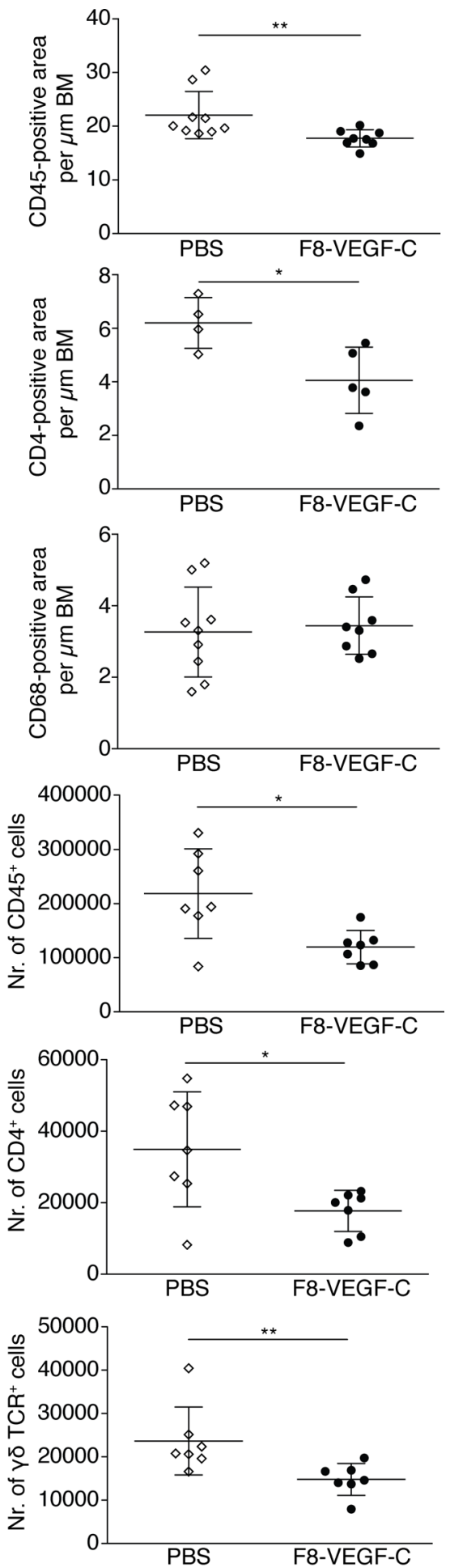

Figure 6. F8-VEGF-C reduces inflammatory cell infiltration in inflamed ears. (A) Immunofluorescence images of inflamed ears of mice that received PBS or F8-VEGF-C stained for CD45 and quantification thereof ( $n=8-9$ animals per group, Mann-Whitney test). (B) Immunofluorescence images of inflamed ears of mice that received PBS or F8-VEGF-C stained for CD4 and quantification thereof ( $n=4-5$ animals per group, 2-tailed Student's $t$ test). (C) Immunofluorescence images of inflamed ears of mice that received PBS or F8-VEGF-C stained for CD68 and quantification thereof ( $n=8-9$ animals per group). (D) Representative flow cytometry plots of live cells in inflamed ears of mice that received PBS (left) or F8-VEGF-C (right) and quantification of absolute numbers of $\mathrm{CD}_{4} 5^{+}$cells ( $n=7$ animals per group, 2-tailed Student's $t$ test with Welch's correction). (E) Representative flow cytometry plots of live cells pregated for CD45 positivity in inflamed ears of mice that received PBS (left) or F8-VEGF-C (right) and quantification of absolute numbers of live CD45/ CD4 double-positive cells ( $n=7$ animals per group, 2-tailed Student's $t$ test with Welch's correction). (F) Representative flow cytometry plots of live cells pregated for CD45 positivity in inflamed ears of mice that received PBS (left) or F8-VEGF-C (right) and quantification of absolute numbers of live CD45/ $\gamma \delta$ T cell receptor double-positive cells ( $n=7$ animals per group, Mann-Whitney test). One of two similar experiments shown. Data represent mean \pm SD. Scale bar: $100 \mu \mathrm{m} .{ }^{*} P<0.05,{ }^{* *} P<0.01$. 
clinically used for the treatment of arthritis and psoriasis, underlining the therapeutic efficacy of the targeted VEGF-C constructs.

Targeted VEGF-C caused a striking expansion of the lymphatic vasculature, in agreement with reports showing an increase in lymphatic vessel number in the skin after local injection (31) or adenoviral delivery of VEGF-C in other disease models $(5,6)$. Untargeted KSF-VEGF-C did not induce lymphatic expansion, highlighting the need for targeted VEGF-C delivery to modify the lymphatic vascular phenotype.

There was no difference between PBS- or SIP-F8- and F8-VEGF-C-treated animals with regards to blood vessel density. Since the fusion protein contains the fully processed $\triangle \mathrm{N} \triangle \mathrm{CVEGF}-\mathrm{C}$ and is able to induce phosphorylation of VEGFR-2 expressed on blood vascular endothelial cells, expansion of the blood vasculature could have been expected and has been reported to occur after adenoviral VEGF-C delivery (32). However, in that study, the angiogenic effects of VEGF-C were limited to high virus titers and appeared to be tissue specific, with skin blood vessels being the least responsive. In line with our findings, K14-VEGF-C mice with constant expression of VEGF-C in the skin do not display changes in the blood vasculature (33). The doses administered in our study over a comparatively short time appear to have been too low to induce changes in blood vessel morphology. Moreover, the induction of blood vessels has been implicated in psoriasis pathogenesis and angiogenesis, and VEGF-A levels correlate with disease severity while blocking VEGF-A alleviated skin inflammation in mouse models of psoriasis $(34,35)$. The observed lack of blood vascular expansion upon treatment with F8-VEGF-C therefore supports the therapeutic potential of the fusion protein.

VEGFR-3 expression has been reported on blood vascular endothelial cells (36) and macrophages (37) under pathological conditions but has previously been demonstrated to be restricted to lymphatic vessels in the K14-VEGF-A model that we used in this study (4). Therefore, VEGFR-3-mediated effects of VEGF-C on these cells types are not to be expected.

Considering that the antiinflammatory effects of F8-VEGF-C and F8-VEGF-C156Ser were comparable in vivo and the nonmutated VEGF-C did not have obvious effects on blood vessels, we focused on the wild-type form of the growth factor.

Clearance of a lymphatic-specific tracer out of the inflamed ears was accelerated significantly in mice injected with F8-VEGF-C compared with animals that had received PBS, indicating that the expanded lymphatic vasculature was fully functional and improved lymphatic drainage. An increase in clearance function in VEGF-C-transgenic mice suffering from chronic inflammation has been reported before (38) and represents one possible mechanism for the fusion protein's antiinflammatory effect. Indeed, K14VEGF-C mice, with their characteristic expanded lymphatic vasculature, show faster antigen clearance in inflamed skin and have an increased inflammatory cell migration to draining lymph nodes (39), which supports our findings of reduced numbers of leukocytes, including CD4 T cells and $\gamma \delta \mathrm{T}$ cells. An improved lymphatic function may increase migration of inflammatory immune cells out of the affected tissue and enhance clearance of antigens and proinflammatory mediators, thereby alleviating inflammation severity. The observed reduction in $\gamma \delta \mathrm{T}$ cells is particularly noteworthy, as these cells have been shown to be the main source of IL-17 in mice, a key cytokine in the establishment and progression of psoriasis, which is largely produced by Th17 cells in humans (40). These $\gamma \delta \mathrm{T}$ cells play a vital role in psoriasis pathogenesis in different disease models. TCR $\delta$ chain-knockout mice undergoing imiquimod-induced inflammation presented with significantly reduced epidermal thickening, neutrophil infiltration, and IL-17 production, while TCR $\alpha$ chain-knockout mice had a normal inflammatory response, indicating that $\gamma \delta \mathrm{T}$ cells are more important than CD4 and/or CD8 T cells in this disease model (28). Furthermore, $\gamma \delta \mathrm{T}$ cells accumulate in the inflamed skin of K14-VEGF-A mice during CHS, and their numbers are increased in the lesional skin of human psoriasis patients (41). In addition, $\gamma \delta \mathrm{T}$ cells have been reported to migrate to draining lymph nodes in the context of skin inflammation, most likely via afferent lymphatic vessels (42). Therefore, a reduction in these disease-driving cells by increased lymphatic drainage may represent a likely mechanism for the antiinflammatory effects of F8-VEGF-C. However, it is important to consider that the relevance of $\gamma \delta \mathrm{T}$ cells in psoriasis pathogenesis in humans is not as clearly established as in mice and other cell populations, e.g., Th17 cells may be the critical players in the human setting.

Apart from increased drainage of proinflammatory immune cells and cytokines, additional mechanisms may be at play. In recent years, research has unveiled the lymphatic endothelium to possess immune-modulating properties. For example, LECs have been shown to secrete immunosuppressive agents such as TGF- $\beta 1$ (38). Furthermore, lymphatic endothelium in lymph nodes displays high levels of 
the T cell inhibitory receptor ligand programmed death-ligand 1 (PD-L1) and lacks costimulatory signals, thus attenuating $\mathrm{T}$ cell activation and inducing peripheral tolerance (43).

In addition, LECs express the decoy receptor D6, which scavenges CCL2 and CCL5, thereby interfering with recruitment of monocytes and $\mathrm{T}$ cells into inflamed tissue (44) and migration of dendritic cells to draining lymph nodes (45). Indeed, mice deficient for D6 failed to clear inflammatory chemokines and suffered from an exacerbated, psoriasis-like skin inflammation (46).

Considering the vast expansion of the lymphatic vasculature, it is possible that a LEC-originating increase in immunosuppressive signaling improves the resolution of inflammation, as observed after treatment with F8-VEGF-C. However, further studies are needed to conclusively elucidate the mechanism underlying the fusion protein's antiinflammatory effects.

Collectively, our work highlights the therapeutic effectiveness of targeted delivery of VEGF-C in the setting of chronic skin inflammation. Unlike viral delivery or local injection of the growth factor, intravenous injection of F8-VEGF-C, followed by targeted accumulation at the inflammation site is a clinically viable application strategy, especially considering that the fusion protein is completely human in sequence, minimizing the risk of immunogenicity in patients. Most likely, targeted VEGF-C could be used to complement available therapeutics aimed at immune cells and cytokines, for example, IL-17A or TNF- $\alpha$ antagonists in the setting of psoriasis.

TNF- $\alpha$ and IL-17 are key cytokines in the pathogenesis of psoriasis. They act on dendritic cells and keratinocytes, triggering the release of a variety of proinflammatory mediators and angiogenic factors (reviewed in ref. 47). Current therapies are aimed at inhibiting TNF- $\alpha$ and IL-17, thus interfering with the inflammatory cascade. In contrast, F8-VEGF-C represents a different therapeutic approach. It does not block proinflammatory signaling directly, but rather activates the lymphatic vasculature, which is an endogenous and physiological supporter of inflammation resolution through a range of mechanisms, e.g., enhanced drainage of immune cells. Due to these mechanistic differences, combining cytokine inhibitors with F8-VEGF-C may improve therapeutic outcomes.

Lymphatic vessels have been reported to persist even after inflammation resolution in the airways and skin $(9,48)$, whereas other studies have demonstrated lymphatic vessel regression, notably in the lymph nodes and in the cornea $(49,50)$. While we have not studied the long-term persistence of the induced lymphatic vessels, it is possible that a comparatively low number of VEGF-C administrations may therefore be sufficient to achieve prolonged antiinflammatory effects.

Based on previous studies and our own preliminary experiments, F8-VEGF-C may represent a viable therapeutic option not only for psoriasis, but for a wide range of chronic inflammatory pathologies, including rheumatoid arthritis (5), chronic inflammatory bowel disease (8), and atherosclerosis, calling for additional research into the therapeutic potential of F8-VEGF-C in these diseases.

\section{Methods}

Cloning of fusion proteins. The genes encoding the F8 (14) or KSF (22) diabody and $\triangle \mathrm{N} \Delta \mathrm{CVEGF-C}$ or $\triangle \mathrm{N} \Delta \mathrm{CVEGF-C156Ser} \mathrm{(20)} \mathrm{were} \mathrm{amplified} \mathrm{from} \mathrm{available} \mathrm{plasmids} \mathrm{encoding} \mathrm{F8-IL4} \mathrm{(51),} \mathrm{KSF,}$ human $\triangle \mathrm{N} \triangle \mathrm{CVEGF-C} \mathrm{(a} \mathrm{gift} \mathrm{of} \mathrm{Kurt} \mathrm{Ballmer-Hofer,} \mathrm{Paul} \mathrm{Scherrer} \mathrm{Institut,} \mathrm{Villigen,} \mathrm{Switzerland),} \mathrm{or}$ $\triangle \mathrm{N} \triangle \mathrm{CVEGF}-\mathrm{C} 156 \mathrm{Ser}$ (a gift of Kari Alitalo, University of Helsinki, Helsinki, Finland) and PCR assembled. The products were digested using HindIII/NotI and cloned into an identically digested pcDNA3.1(+) plasmid backbone (Invitrogen).

Expression of fusion proteins. The fusion proteins were expressed in FreeStyle CHO-S cells (Thermo Fisher) using transient gene expression. Briefly, cells were cultured in suspension in PowerCHO-2CD medium (Lonza) and supplemented with HT supplement containing $100 \mu \mathrm{M}$ hypoxanthine, $16 \mu \mathrm{M}$ thymidine (Lonza), and $2 \mathrm{mM}$ L-glutamine (Life Technologies). For transfection, cells were transferred into ProCHO-4 medium (Lonza); supplemented with HT supplement containing $100 \mu \mathrm{M}$ hypoxanthine, $16 \mu \mathrm{M}$ thymidine, and $2 \mathrm{mM}$ L-glutamine; and plasmid premixed with polyethylenimine (PEI) was added (DNA/ PEI ratio of $1: 4)$. Cells were incubated at $37^{\circ} \mathrm{C}$ and PowerCHO-2CD was added $1: 1$ approximately $3-4$ hours after transfection. Twenty-hour hours later, the incubation temperature was lowered to $32^{\circ} \mathrm{C}$ and cells were cultured for 5-7 days at an initial cell density of $1 \times 10^{6}$ cells per ml.

In the case of F8-VEGF-C and F8-VEGF-C156Ser, stably transfected monoclonal cell lines were established using serial dilution of transiently transfected CHO-S cells kept under G-418 (Life Technologies) selection for $>28$ days. 
In the case of KSF-VEGF-C, stably transfected monoclonal cell lines were established by electroporation (Nucleofector V kit, Lonza, used according to the manufacturer's instructions), followed by G-418 (Roche) selection for $>28$ days and fluorescence-activated cell sorting of cells stained with rabbit anti-human IgG (A0423, Dako) and Alexa Fluor 488-conjugated goat anti-rabbit secondary antibody (Invitrogen) on a FACSAria IIu (BD Biosciences) using FACSDiva software (BD Biosciences).

Protein purification and size exclusion chromatography. The fusion proteins were purified by protein A affinity chromatography (HiTrap Protein A HP, GE Healthcare Life Sciences), essentially as described previously (52). Purified fusion proteins were analyzed by size exclusion chromatography on a Superdex200 10/300GL column (GE Healthcare).

Immunofluorescence. OCT-embedded ear samples of K14-VEGF-A-transgenic and imiquimod-treated mice were frozen on liquid nitrogen, and 7- $\mu \mathrm{m}$ cryostat sections were prepared. After fixation in acetone and rehydration in 80\% methanol, the sections were blocked with PBS containing 5\% donkey serum, $0.1 \%$ Triton-X, and $1 \%$ bovine serum albumin, followed by incubation with the respective primary antibodies (as listed below). Human samples (healthy and psoriatic skin) were processed identically, except for fixation, which was done in Fixation/Permeabilization buffer (eBioscience). Standard immunofluorescence stainings were performed as described previously $(4,18)$, using the following antibodies: biotinylated F8 (provided in-house), polyclonal goat anti-human VE-cadherin (Santa Cruz Biotechnology), mouse anti-human EDA (IST-9, Abcam), polyclonal rabbit anti-mouse LYVE-1 (Angiobio), rat anti-mouse CD31 (MEC13.3, BD Pharmingen), rat anti-mouse MECA-32 (BD Biosciences), polyclonal goat anti-mouse CD45 (R\&D Systems), and rat anti-mouse CD4 (GK1.5, eBioscience). Alexa Fluor 488- and Alexa Fluor 594-conjugated secondary antibodies and Hoechst 33342 were purchased from Invitrogen, and Alexa Fluor 594-conjugated streptavidin was purchased from Life Technologies. Slides were mounted with Mowiol mounting medium.

Morphometric analyses. Tissue sections were imaged on an Axioskop2 mot plus microscope (Carl Zeiss) with an AxioCam MRc camera (Carl Zeiss) and a Plan-APOCHROMAT $\times 10$ or $\times 20$ objective (Carl Zeiss). Using AxioVision software 4.8 (Carl Zeiss), at least 3 fields of view were acquired per sample. Regions of interest were defined as the area of one ear half between stratum corneum and central cartilage. The positive area of interest was measured or vessels were counted using ImageJ $(\mathrm{NIH})$ software version $1.49 \mathrm{v}$ in a blinded fashion. Results are expressed as positive area or vessel count normalized to $\mu \mathrm{m}$ or mm of basement membrane, as inflammatory edema causes an increase in tissue area, which might confound results normalized to area.

For the diaphragm assay, Z-stack images were acquired with a Zeiss LSM 710-FCS confocal microscope (Carl Zeiss) equipped with a $\times 100.3$ NA EC Plan-Neofluar objective, using the Zeiss ZEN software. Regions of interest were defined as the diaphragmatic muscle, and positive area of interest was calculated with ImageJ software version $1.49 \mathrm{v}$ in a blinded fashion and expressed as percentage of area.

Western blots. Proteins were analyzed by SDS-PAGE using 4\%-12\% Bis-Tris gels (NuPAGE system, Life Technologies) and Coomassie staining or Western blots. Proteins were transferred to PVDF membranes (Invitrogen) and incubated with polyclonal rabbit anti-VEGF-C antibody (ab9546, Abcam), followed by HRP-conjugated secondary antibody [donkey ECL anti-rabbit IgG, HRP-linked $\mathrm{F}\left(\mathrm{ab}^{\prime}\right)_{2}$ fragment, GE Healthcare]. Signals were visualized by chemiluminescence (ECL Prime, Amersham) on an Agfa Curix 60 developer.

Phosphorylation analyses. For VEGFR-3 phosphorylation analysis, PAE cells, which overexpress VEGFR-3 (23) (a gift of Lena Claesson-Welsh, Uppsala University, Uppsala, Sweden), were stimulated for 20 minutes with VEGF-C (200 ng/ml), VEGF-C156Ser (200 ng/ml), F8-VEGF-C (corresponding to $200 \mathrm{ng} / \mathrm{ml}$ VEGF-C), and F8-VEGF-C156Ser (corresponding to $200 \mathrm{ng} / \mathrm{ml} \mathrm{VEGF-C156Ser)} \mathrm{and}$ then lysed in RIPA buffer (50 mM Tris- $\mathrm{HCl}$ [pH 7.5], $150 \mathrm{mM} \mathrm{NaCl}, 0.5 \%$ NP-40, $5 \mathrm{mM}$ EDTA, 1\% Triton-X100, and $\left.1 \mathrm{mM} \mathrm{Na} \mathrm{VO}_{4}\right)$. VEGFR-3 was immunoprecipitated by using Dynabeads Protein G (Invitrogen) complexed with anti-VEGFR-3 antibody (C20, Santa Cruz Biotechnology). Immunoprecipitated samples were subjected to Western blot using an anti-phosphotyrosine antibody (4G10, Millipore). Subsequently, the membrane was stripped for 30 minutes in stripping buffer containing Tris, SDS, and 2-mercaptoethanol and reprobed with VEGFR-3 antibody (C20, Santa Cruz Biotechnology). To investigate VEGFR-2 phosphorylation, human LECs were stimulated and processed as described above. Following stripping, the membrane was reprobed with anti-human VEGFR-2 antibody (Cell Signaling).

Surface plasmon resonance analyses. Affinities of VEGF-C fusion proteins were measured on a Biacore S200 (GE Healthcare) surface plasmon resonance sensor. Different dilutions of fusion proteins in filtered $(0.44 \mu \mathrm{m})$ PBS (500 nM, $250 \mathrm{nM}, 125 \mathrm{nM}, 62.5 \mathrm{nM}$, and $32.25 \mathrm{nM}$ ) were recorded in order of ascending 
concentrations on an EDA-coated gold chip. Quantification was performed with Biacore S200 Evaluation Software (GE Healthcare, version 1.0).

Proliferation assays. Proliferation assays were performed as described previously (53). Briefly, 2500 human LECs were seeded into collagen-coated 96-well plates in EBM medium (Lonza) containing 20\% FBS (Gibco), $25 \mu \mathrm{g} / \mathrm{ml}$ cAMP (MilliporeSigma) and $10 \mu \mathrm{g} / \mathrm{ml}$ hydrocortisone (MilliporeSigma). After an overnight starvation in EBM containing 0.5\% FBS, the cells were incubated with VEGF-A $(20 \mathrm{ng} / \mathrm{ml}$, Cell Sciences), VEGF-C (200 ng/ml, gift of Kurt Ballmer-Hofer), F8-diabody (400 ng/ml), KSF-VEGF-C (600 ng/ml), F8-VEGF-C (600 ng/ml), or F8-VEGF-C156Ser (600 ng/ml). After 72 hours, $100 \mu \mathrm{g} / \mathrm{ml}$ 4-methylumbelliferyl heptanoate (MilliporeSigma) was added, and the fluorescence intensity, corresponding to the number of viable cells (54), was measured on a SpectraMax reader (Molecular Devices) at $355 \mathrm{~nm}$ excitation and $460 \mathrm{~nm}$ emission. For each condition, at least quintuplicates were analyzed and the assay was performed 3 times.

Sprouting assay. The 3-dimensional sprouting assay was performed as described previously (55). Briefly, LEC-coated cyclodextran microcarrier beads (MilliporeSigma) were labeled with cell tracker green (Invitrogen) and embedded in collagen type I hydrogels $(1 \mathrm{mg} / \mathrm{ml}$, Advanced Bio-Matrix), followed by the addition of LEC medium (EBM, 20\% FBS, and $40 \mathrm{ng} / \mathrm{ml}$ bFGF [R\&D Systems]). VEGF-A (40 ng/ml), VEGF-C (200 ng/ml), VEGF-C156Ser (200 ng/ml), F8-VEGF-C (corresponding to $200 \mathrm{ng} / \mathrm{ml}$ VEGF-C), F8-VEGF-C156Ser (corresponding to $200 \mathrm{ng} / \mathrm{ml}$ VEGF-C156Ser), or SIP-F8 (identical dose as F8-VEGF-C/ F8-VEGF-C156Ser) were added to the medium. After 24 hours at $37^{\circ} \mathrm{C}$, imaging was conducted and sprouts were counted manually. For each condition, quintuplicates were analyzed and the assay was performed twice.

Diaphragm lymphangiogenesis assay. In order to study the effects of F8-VEGF-C and KSF-VEGF-C on lymphatic vessels in the diaphragm, neonatal FVB mice received daily intraperitoneal injections of F8-VEGF-C (2.27 $\mu \mathrm{g} / \mathrm{g}$ body weight), KSF-VEGF-C (2.27 $\mu \mathrm{g} / \mathrm{g}$ body weight), or PBS on days P1-P5. Mice were euthanized on P6 and processed as described previously (56). Briefly, diaphragms were excised and fixed in 4\% PFA for 2 hours, followed by blocking in 5\% donkey serum, $0.1 \%$ Triton-X, and $1 \%$ BSA in PBS for 1 hour. The samples were then incubated with primary antibodies overnight at $4^{\circ} \mathrm{C}$. Primary antibodies used were polyclonal rabbit anti-mouse LYVE-1 (Angiobio) and rat anti-mouse CD31 (MEC13.3, BD Pharmingen). Alexa Fluor 488- and 594-conjugated secondary antibodies were purchased from Invitrogen. Stained samples were flat-mounted on glass slides.

Serum half-life determination. After tail vein injection of $50 \mu \mathrm{g}$ F8-VEGF-C fusion protein, serial blood samples were taken from the saphenous vein at 2 minutes, 10 minutes, 30 minutes, 1 hour, and 4 hours. After 20 minutes of incubation at room temperature, the blood samples were centrifuged at $1200 \mathrm{~g}$ for 10 minutes. The sera were collected, snap frozen, and stored at $-80^{\circ} \mathrm{C}$. The amount of F8-VEGF-C within each serum sample was determined using the human VEGF-C Quantikine ELISA Kit (R\&D Systems) according to the manufacturer's protocol.

Mouse models. The mice used in this study were housed in the animal facility of ETH Zurich under specific pathogen-free conditions.

K14-VEGF-A-transgenic mice have been described previously $(30,57)$. We used female hemizygous K14-VEGF-A mice on the FVB background, aged 8-9 weeks at the beginning of the study, at least 5 animals per group; all were bred and housed in the animal facility of ETH Zürich. Briefly, sensitization by a topical application of a $2 \%$ oxazolone solution (4-ethoxymethylene-2 phenyl-2-oxazoline-5-one; MilliporeSigma) in acetone/olive oil $(4: 1 \mathrm{~V} / \mathrm{V})$ on the shaved abdomen $(50 \mu \mathrm{l})$ and on each paw $(5 \mu \mathrm{l})$, followed by a topical application of $10 \mu \mathrm{l}$ oxazolone (1\%) on each side of both ears 5 days later (challenge), led to a chronic skin inflammation in these mice (18). Ear thickness was measured before and repeatedly after challenge using calipers. Starting on day 7, the following treatments were applied intravenously every second day, until day 13 (2 days before termination): PBS, SIP-F8 (50 $\mu$ g), TNFR-Fc (30 $\mu$ g, ref. 25), or F8-VEGF-C, F8-VEGF-C156Ser, or KSF-VEGF-C (50 $\mu$ g of each construct). On day 15, the mice were euthanized.

In a second model of skin inflammation, an imiquimod-containing cream (Aldara, 3M Pharmaceuticals) was applied daily to the ear skin of 8-week-old female C57BL/ 6 wild-type mice obtained from Janvier Labs (at least 5 per group) for 5 consecutive days, followed by 1 day without treatment and another imiquimod dose on day 7. Starting on day 7, each mouse received every second day intravenous injections of PBS, SIP-F8, TNFR-Fc, F8-VEGF-C, F8-VEGF-C156Ser, or KSF-VEGF-C, with the last injection on day 11. Ear thickness was measured daily. The mice were euthanized on day 12 or 13.

Biodistribution analyses. $15 \mu \mathrm{g}$ radioiodinated F8-VEGF-C, F8-VEGF-C156Ser, or the control SIP-F8 were injected intravenously into the lateral tail vein of inflamed or uninflamed mice (for details on the labeling 
protocol see ref. 58). To evaluate the targeting properties in the two models of cutaneous inflammation, both K14-VEGF-A-transgenic mice on day 7 after challenge and imiquimod-treated C57BL/ 6 wild-type mice after 7 days of continuous application of imiquimod (no treatment on day 6 as indicated above) as well as uninflamed FVB and BL6 wild-type mice were subjected to this analysis. Twenty-four hours after the injection, the organs were dissected and weighted and the radioactivity was measured by a Packard Cobra $\gamma$ counter. The radioactivity of individual organs is expressed as the percentage of injected dose per gram of tissue (\%ID/g).

Flow cytometry. Ears were harvested from K14-VEGF-A-transgenic mice on day 15 after oxazolone challenge. Following mechanical disruption of the tissue, samples were digested under rotation in DMEM (Gibco) containing $4 \mathrm{mg} / \mathrm{ml}$ collagenase IV (Gibco) at $37^{\circ} \mathrm{C}$ for 45 minutes. Samples were filtered through $40-\mu \mathrm{m}$ strainers (Falcon), centrifuged, and resuspended in FACS buffer containing 2\% FBS (Gibco) and 2 mM EDTA (Fluka). Samples were incubated with anti-mouse CD16/32 antibody (93, Biolegend) on ice for 20 minutes and subsequently stained on ice for 30 minutes using Pacific Blue anti-mouse CD45 (30-F11, Biolegend), APC anti-mouse CD4 (GK1.5, Biolegend), FITC anti-mouse CD8 (53-6.7, Biolegend), and PerCP-eFluor 710 anti-mouse $\gamma \delta$ TCR (GL-3, eBioscience) antibodies and live/dead fixable aqua dye (Life Technologies). Samples were acquired on an LSRFortessa (BD Biosciences) using FACSDiva software. Analysis was performed using FlowJo v10.1 in a blinded fashion. Quantification of absolute cell numbers was done using AccuCheck counting beads (Invitrogen).

Clearance assay. The polyethylene glycol-based (PEG-based) lymphatic tracer P20D800 (PEG amine P20 conjugated to IRDye (LI-COR Biosciences) was prepared as described previously (59). To examine lymphatic clearance over time, inflamed K14-VEGF-A-transgenic mice (10 days after oxazolone challenge) were anesthetized with isoflurane $(2 \%)$, and $3 \mu \mathrm{l}$ of $3 \mu \mathrm{M}$ tracer was injected intradermally into the ear skin. The mice were positioned in an IVIS Spectrum imaging system, and an image was acquired just after tracer injection, with an exposure of 2 seconds $\left(\lambda_{\mathrm{ex}}: 745 \mathrm{~nm}, \lambda_{\mathrm{em}}: 800 \mathrm{~nm}\right.$, binning of 4), and then 1 hour, 2 hours, 3 hours, 4 hours, and 6 hours after the injection. Between the different imaging time points, mice were allowed to wake up and move freely. In order to calculate tissue enhancement values, all signal intensities were adjusted to baseline ear signals before tracer injection. The tissue enhancement value obtained directly after the injection of the tracer was used to normalize all values of the subsequent measurements. A 1-phase exponential decay model was used (27) with equations provided later in this sentence to fit the data for each mouse, with lymphatic clearance expressed as decay constant $K$ (expressed in $\mathrm{h}^{-1}$ ) or as half-life (expressed in h): (a) normalized tissue enhancement $=e^{-K t}$ and (b) half-life $=\ln (2 / K)$, where $\ln$ is the natural logarithm, $e$ is Euler's number, $K$ is the decay constant, and $t$ is time.

Statistics. Statistical analyses were performed using Prism version 7.0a (GraphPad Software Inc.). Data are shown as mean $\pm \mathrm{SD}$. To determine statistical significance, a 2-tailed, unpaired Student's $t$ test (for the comparison of 2 groups), a Mann-Whitney test (in case Shapiro-Wilk test showed data to not be normally distributed), a $t$ test with Welch's correction (if the variances of 2 groups to be compared differ significantly), a 1-way ANOVA (for the comparison of more than 2 groups), or a 2-way ANOVA (for repeated measurements) with Bonferroni post test or Games-Howell post test (in case variances differed significantly between groups) were performed. Samples failing a Grubbs' outlier test were excluded. Differences were considered statistically significant at $P \leq 0.05$.

Study approval. Human studies were approved by the local ethics committee (Kantonale Ethikkommission Zürich, approval 2017-00687), and informed consent was obtained from participants. Studies involving mice were performed in accordance with animal protocols (117/2011, 37/2013, and 212/2016) approved by the local veterinary authorities (Kantonales Veterinäramt Zürich, Zurich, Switzerland.

\section{Author contributions}

SS and SR designed the research, performed experiments, analyzed results, and wrote the manuscript. TH, STP, SK, RF, AMGO, and PP performed experiments and analyzed results. DN and $\mathrm{CH}$ designed the research and analyzed results. MD designed the research, analyzed results, and wrote the manuscript.

\section{Acknowledgments}

This study was supported by Swiss National Science Foundation grants 310030B_147087 and 310030_166490 and Advanced European Research Council grant LYVICAM (to MD) as well as by Swiss National Science Foundation grant 310030B_163479/1 and Advanced European Research Council Grant ZAUBERKUGEL (to $\mathrm{DN})$. The authors are grateful to Kurt Ballmer-Hofer for the plasmid encoding $\triangle \mathrm{N} \Delta \mathrm{CVEGF}-\mathrm{C}$, Kari Alitalo for 
the plasmid encoding $\triangle \mathrm{N} \triangle \mathrm{CVEGF-C156Ser}$, and Lena Claesson-Welsh for the VEGFR-3-overexpressing PAE cells. Furthermore, the authors thank Samia Bachmann, Marco D'Addio, Epameinondas Gousopoulos, Jeannette Scholl, and Marko Sesartic for excellent technical assistance; Carlos Ochoa, Sven Nowok, Marion Hermerschmidt, and Dennis Mollenhauer for help with the animal studies; the ETH Flow Cytometry Core Facility for assistance with flow cytometry; and the Scientific Center for Optical and Electron Microscopy (ScopeM) of ETH Zurich for assistance with confocal imaging.

Address correspondence to: Michael Detmar, Institute of Pharmaceutical Sciences, Swiss Federal Institute of Technology, ETH Zurich, Vladimir-Prelog-Weg 3, HCI H303, CH-8093 Zurich, Switzerland. Phone: 41.44.633.7361; Email: michael.detmar@pharma.ethz.ch.

SR's present address is: MSD Merck Sharp and Dohme AG, Lucerne, Switzerland.

TH's present address is: Philochem AG, Philogen Group, Otelfingen, Switzerland.

SK's present address is: Wihuri Research Institute, Translational Cancer Biology, Helsinki, Finland.

AMGO's present address is: Novartis Institutes for Biomedical Research, Basel, Switzerland.

1. Alitalo K. The lymphatic vasculature in disease. Nat Med. 2011;17(11):1371-1380.

2. Dieterich LC, Seidel CD, Detmar M. Lymphatic vessels: new targets for the treatment of inflammatory diseases. Angiogenesis. 2014;17(2):359-371

3. Kaipainen A, et al. Expression of the fms-like tyrosine kinase 4 gene becomes restricted to lymphatic endothelium during development. Proc Natl Acad Sci USA. 1995;92(8):3566-3570.

4. Huggenberger R, Ullmann S, Proulx ST, Pytowski B, Alitalo K, Detmar M. Stimulation of lymphangiogenesis via VEGFR-3 inhibits chronic skin inflammation. J Exp Med. 2010;207(10):2255-2269.

5. Zhou Q, et al. Vascular endothelial growth factor $\mathrm{C}$ attenuates joint damage in chronic inflammatory arthritis by accelerating local lymphatic drainage in mice. Arthritis Rheum. 2011;63(8):2318-2328.

6. D'Alessio S, et al. VEGF-C-dependent stimulation of lymphatic function ameliorates experimental inflammatory bowel disease. J Clin Invest. 2014;124(9):3863-3878.

7. Guo R, et al. Inhibition of lymphangiogenesis and lymphatic drainage via vascular endothelial growth factor receptor 3 blockade increases the severity of inflammation in a mouse model of chronic inflammatory arthritis. Arthritis Rheum. 2009;60(9):2666-2676

8. Jurisic G, Sundberg JP, Detmar M. Blockade of VEGF receptor-3 aggravates inflammatory bowel disease and lymphatic vessel enlargement. Inflamm Bowel Dis. 2013;19(9):1983-1989.

9. Baluk P, et al. Pathogenesis of persistent lymphatic vessel hyperplasia in chronic airway inflammation. J Clin Invest. 2005;115(2):247-257.

10. Lieberman JR, Daluiski A, Einhorn TA. The role of growth factors in the repair of bone. Biology and clinical applications. J Bone Joint Surg Am. 2002;84-A(6):1032-1044.

11. Henry TD, et al. The VIVA trial: Vascular endothelial growth factor in ischemia for vascular angiogenesis. Circulation. 2003;107(10):1359-1365.

12. Young PA, Morrison SL, Timmerman JM. Antibody-cytokine fusion proteins for treatment of cancer: engineering cytokines for improved efficacy and safety. Semin Oncol. 2014;41(5):623-636.

13. Bootz F, Neri D. Immunocytokines: a novel class of products for the treatment of chronic inflammation and autoimmune conditions. Drug Discov Today. 2016;21(1):180-189.

14. Villa A, et al. A high-affinity human monoclonal antibody specific to the alternatively spliced EDA domain of fibronectin efficiently targets tumor neo-vasculature in vivo. Int J Cancer. 2008;122(11):2405-2413.

15. Hemmerle T, Zgraggen S, Matasci M, Halin C, Detmar M, Neri D. Antibody-mediated delivery of interleukin 4 to the neo-vasculature reduces chronic skin inflammation. J Dermatol Sci. 2014;76(2):96-103.

16. Bootz F, Schmid AS, Neri D. Alternatively spliced EDA domain of fibronectin is a target for pharmacodelivery applications in inflammatory bowel disease. Inflamm Bowel Dis. 2015;21(8):1908-1917.

17. Hemmerle T, Doll F, Neri D. Antibody-based delivery of IL4 to the neovasculature cures mice with arthritis. Proc Natl Acad Sci USA. 2014;111(33):12008-12012.

18. Kunstfeld R, et al. Induction of cutaneous delayed-type hypersensitivity reactions in VEGF-A transgenic mice results in chronic skin inflammation associated with persistent lymphatic hyperplasia. Blood. 2004;104(4):1048-1057

19. van der Fits L, et al. Imiquimod-induced psoriasis-like skin inflammation in mice is mediated via the IL-23/IL-17 axis. J Immunol. 2009;182(9):5836-5845.

20. Joukov V, et al. A recombinant mutant vascular endothelial growth factor-C that has lost vascular endothelial growth factor receptor-2 binding, activation, and vascular permeability activities. J Biol Chem. 1998;273(12):6599-6602.

21. Holliger P, Hudson PJ. Engineered antibody fragments and the rise of single domains. Nat Biotechnol. 2005;23(9):1126-1136.

22. Frey K, Zivanovic A, Schwager K, Neri D. Antibody-based targeting of interferon-alpha to the tumor neovasculature: a critical evaluation. Integr Biol (Camb). 2011;3(4):468-478. 
23. Dixelius J, et al. Ligand-induced vascular endothelial growth factor receptor-3 (VEGFR-3) heterodimerization with VEGFR-2 in primary lymphatic endothelial cells regulates tyrosine phosphorylation sites. J Biol Chem. 2003;278(42):40973-40979.

24. Van Belle AB, et al. IL-22 is required for imiquimod-induced psoriasiform skin inflammation in mice. J Immunol. 2012;188(1):462-469.

25. Doll F, Schwager K, Hemmerle T, Neri D. Murine analogues of etanercept and of F8-IL10 inhibit the progression of collagen-induced arthritis in the mouse. Arthritis Res Ther. 2013;15(5):R138.

26. Proulx ST, et al. Non-invasive dynamic near-infrared imaging and quantification of vascular leakage in vivo. Angiogenesis. 2013;16(3):525-540.

27. Karaman S, Buschle D, Luciani P, Leroux JC, Detmar M, Proulx ST. Decline of lymphatic vessel density and function in murine skin during aging. Angiogenesis. 2015;18(4):489-498.

28. Cai Y, et al. Pivotal role of dermal IL-17-producing $\gamma \delta$ T cells in skin inflammation. Immunity. 2011;35(4):596-610.

29. Schwager K, et al. Preclinical characterization of DEKAVIL (F8-IL10), a novel clinical-stage immunocytokine which inhibits the progression of collagen-induced arthritis. Arthritis Res Ther. 2009;11(5):R142.

30. Xia YP, Li B, Hylton D, Detmar M, Yancopoulos GD, Rudge JS. Transgenic delivery of VEGF to mouse skin leads to an inflammatory condition resembling human psoriasis. Blood. 2003;102(1):161-168.

31. Kajiya K, Sawane M, Huggenberger R, Detmar M. Activation of the VEGFR-3 pathway by VEGF-C attenuates UVB-induced edema formation and skin inflammation by promoting lymphangiogenesis. J Invest Dermatol. 2009;129(5):1292-1298

32. Saaristo A, et al. Adenoviral VEGF-C overexpression induces blood vessel enlargement, tortuosity, and leakiness but no sprouting angiogenesis in the skin or mucous membranes. FASEB J. 2002;16(9):1041-1049.

33. Jeltsch M, et al. Hyperplasia of lymphatic vessels in VEGF-C transgenic mice. Science. 1997;276(5317):1423-1425.

34. Detmar M, et al. Overexpression of vascular permeability factor/vascular endothelial growth factor and its receptors in psoriasis. J Exp Med. 1994;180(3):1141-1146

35. Schonthaler HB, Huggenberger R, Wculek SK, Detmar M, Wagner EF. Systemic anti-VEGF treatment strongly reduces skin inflammation in a mouse model of psoriasis. Proc Natl Acad Sci USA. 2009;106(50):21264-21269.

36. Paavonen K, Puolakkainen P, Jussila L, Jahkola T, Alitalo K. Vascular endothelial growth factor receptor-3 in lymphangiogenesis in wound healing. Am J Pathol. 2000;156(5):1499-1504.

37. Zhang Y, et al. Activation of vascular endothelial growth factor receptor-3 in macrophages restrains TLR4-NF- $\mathrm{kB}$ signaling and protects against endotoxin shock. Immunity. 2014;40(4):501-514.

38. Christiansen AJ, et al. Lymphatic endothelial cells attenuate inflammation via suppression of dendritic cell maturation. Oncotar get. 2016;7(26):39421-39435

39. Kataru RP, et al. Critical role of CD11b+ macrophages and VEGF in inflammatory lymphangiogenesis, antigen clearance, and inflammation resolution. Blood. 2009;113(22):5650-5659.

40. Pantelyushin S, et al. Ror $\gamma \mathrm{t}+$ innate lymphocytes and $\gamma \delta \mathrm{T}$ cells initiate psoriasiform plaque formation in mice. $J$ Clin Invest. 2012;122(6):2252-2256

41. Suzuki T, et al. VEGF-A promotes IL-17A-producing $\gamma \delta \mathrm{T}$ cell accumulation in mouse skin and serves as a chemotactic factor for plasmacytoid dendritic cells. J Dermatol Sci. 2014;74(2):116-124.

42. Saito T, Yano M, Ohki Y, Tomura M, Nakano N. Occludin expression in epidermal $\gamma \delta$ T cells in response to epidermal stress causes them to migrate into draining lymph nodes. J Immunol. 2017;199(1):62-71.

43. Tewalt EF, et al. Lymphatic endothelial cells induce tolerance via PD-L1 and lack of costimulation leading to high-level PD-1 expression on CD8 T cells. Blood. 2012;120(24):4772-4782.

44. Ajuebor MN, et al. Endogenous monocyte chemoattractant protein-1 recruits monocytes in the zymosan peritonitis model. J Leukoc Biol. 1998;63(1):108-116.

45. Sato N, et al. CC chemokine receptor (CCR)2 is required for langerhans cell migration and localization of $\mathrm{T}$ helper cell type 1 (Th1)-inducing dendritic cells. Absence of CCR2 shifts the Leishmania major-resistant phenotype to a susceptible state dominated by Th2 cytokines, b cell outgrowth, and sustained neutrophilic inflammation. J Exp Med. 2000;192(2):205-218.

46. Jamieson T, et al. The chemokine receptor D6 limits the inflammatory response in vivo. Nat Immunol. 2005;6(4):403-411.

47. Hawkes JE, Chan TC, Krueger JG. Psoriasis pathogenesis and the development of novel targeted immune therapies. $J$ Allergy Clin Immunol. 2017;140(3):645-653.

48. Nagy JA, et al. Vascular permeability factor/vascular endothelial growth factor induces lymphangiogenesis as well as angiogenesis. J Exp Med. 2002;196(11):1497-1506.

49. Mumprecht V, Roudnicky F, Detmar M. Inflammation-induced lymph node lymphangiogenesis is reversible. Am J Pathol. 2012;180(3):874-879.

50. Kelley PM, Connor AL, Tempero RM. Lymphatic vessel memory stimulated by recurrent inflammation. Am J Pathol. 2013;182(6):2418-2428

51. Hemmerle T, Neri D. The antibody-based targeted delivery of interleukin- 4 and 12 to the tumor neovasculature eradicates tumors in three mouse models of cancer. Int J Cancer. 2014;134(2):467-477.

52. Villa A, Lovato V, Bujak E, Wulhfard S, Pasche N, Neri D. A novel synthetic naïve human antibody library allows the isolation of antibodies against a new epitope of oncofetal fibronectin. MAbs. 2011;3(3):264-272

53. Hirakawa $\mathrm{S}$, et al. Identification of vascular lineage-specific genes by transcriptional profiling of isolated blood vascular and lymphatic endothelial cells. Am J Pathol. 2003;162(2):575-586.

54. Stadler R, Detmar M, Stephanek K, Bangemann C, Orfanos CE. A rapid fluorometric assay for the determination of keratinocyte proliferation in vitro. J Invest Dermatol. 1989;93(4):532-534.

55. Schulz MM, et al. Phenotype-based high-content chemical library screening identifies statins as inhibitors of in vivo lymphangiogenesis. Proc Natl Acad Sci USA. 2012;109(40):E2665-E2674.

56. Ochsenbein AM, et al. Regulation of lymphangiogenesis in the diaphragm by macrophages and VEGFR-3 signaling. Angiogenesis. 2016;19(4):513-524.

57. Detmar M, et al. Increased microvascular density and enhanced leukocyte rolling and adhesion in the skin of VEGF transgenic mice. J Invest Dermatol. 1998;111(1):1-6. 
58. Pasche N, Woytschak J, Wulhfard S, Villa A, Frey K, Neri D. Cloning and characterization of novel tumor-targeting immunocytokines based on murine IL7. J Biotechnol. 2011;154(1):84-92.

59. Proulx ST, et al. Use of a PEG-conjugated bright near-infrared dye for functional imaging of rerouting of tumor lymphatic drainage after sentinel lymph node metastasis. Biomaterials. 2013;34(21):5128-5137. 